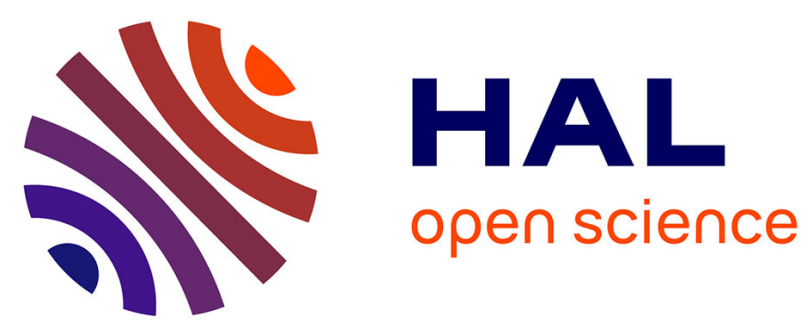

\title{
A projection algorithm for gradient waveforms design in Magnetic Resonance Imaging
}

\author{
Nicolas Chauffert, Pierre Weiss, Jonas Kahn, Philippe Ciuciu
}

\section{To cite this version:}

Nicolas Chauffert, Pierre Weiss, Jonas Kahn, Philippe Ciuciu. A projection algorithm for gradient waveforms design in Magnetic Resonance Imaging. IEEE Transactions on Medical Imaging, 2016, 35

(9), pp.2026-2039. 10.1109/TMI.2016.2544251 . hal-01317939

\section{HAL Id: hal-01317939 \\ https://hal.inria.fr/hal-01317939}

Submitted on 20 May 2016

HAL is a multi-disciplinary open access archive for the deposit and dissemination of scientific research documents, whether they are published or not. The documents may come from teaching and research institutions in France or abroad, or from public or private research centers.
L'archive ouverte pluridisciplinaire HAL, est destinée au dépôt et à la diffusion de documents scientifiques de niveau recherche, publiés ou non, émanant des établissements d'enseignement et de recherche français ou étrangers, des laboratoires publics ou privés. 


\title{
A projection algorithm for gradient waveforms design in Magnetic Resonance Imaging
}

\author{
Nicolas Chauffert, Pierre Weiss, Jonas Kahn and Philippe Ciuciu Senior Member
}

\begin{abstract}
Collecting the maximal amount of information in a given scanning time is a major concern in Magnetic Resonance Imaging (MRI) to speed up image acquisition. The hardware constraints (gradient magnitude, slew rate, ...), physical distortions (e.g., off-resonance effects) and sampling theorems (Shannon, compressed sensing) must be taken into account simultaneously, which makes this problem extremely challenging. To date, the main approach to design gradient waveform has consisted of selecting an initial shape (e.g. spiral, radial lines, ...) and then traversing it as fast as possible using optimal control.

In this paper, we propose an alternative solution which first consists of defining a desired parameterization of the trajectory and then of optimizing for minimal deviation of the sampling points within gradient constraints. This method has various advantages. First, it better preserves the density of the input curve which is critical in sampling theory. Second, it allows to smooth high curvature areas making the acquisition time shorter in some cases. Third, it can be used both in the Shannon and CS sampling theories. Last, the optimized trajectory is computed as the solution of an efficient iterative algorithm based on convex programming. For piecewise linear trajectories, as compared to optimal control reparameterization, our approach generates a gain in scanning time of $10 \%$ in echo planar imaging while improving image quality in terms of signal-to-noise ratio (SNR) by more than $6 \mathrm{~dB}$. We also investigate original trajectories relying on traveling salesman problem solutions. In this context, the sampling patterns obtained using the proposed projection algorithm are shown to provide significantly better reconstructions (more than $6 \mathrm{~dB}$ ) while lasting the same scanning time.
\end{abstract}

\section{Keywords}

gradient waveform design, $k$-space trajectories, variable density sampling, gradient hardware constraints, magnetic resonance imaging.

\section{INTRODUCTION}

$\mathbf{T}$ HE advent of new hardware and sampling theories (e.g., Compressed Sensing or CS) provide unprecedented opportunities to reduce acquisition times in MRI. The design of gradient waveforms minimizing the acquisition time while providing enough information to reconstruct distortion-free images is however an important challenge. Ideally, these two concerns (sampling scheme and gradient waveform design) should be addressed simultaneously, but current theoretical results in sampling theories (either Shannon-based or CS-based) do not permit to incorporate complex physical constraints like the starting position or the traversal speed in $k$-space, despite recent progresses [1-5].

To date, the most widespread technique therefore consists of designing gradient waveforms sequentially: a first step aims to find the trajectory support or at least control points, and a second step builds the gradient waveforms to traverse this support or to link these control points. The first step either relies on Shannon sampling theorem $[4,5]$ or on the concept of variable density sampling (VDS) [2,6,7]. In Shannon theory, the samples located in the $k$-space should lie on a Cartesian grid with a sufficiently small grid step size. A typical instance of such schemes is the echo planar imaging (EPI) trajectory. The wealth of trajectories in VDS is constantly increasing and becomes more and more anchored in theory. It initially started with spirals [8, 9] and was progressively enriched with different patterns such as parallel or radial lines [10,11], noisy spirals [12], Rosette trajectories [13], shell trajectories [14], ... The second step is currently solved by using reparameterization: the goal is to find a feasible waveform traversing the support in the minimum amount of time. This problem can be solved using optimal control [15], convex optimization [16,17], or optimal interpolation of $k$-space control points [18]. These simple principles however suffer from potentially severe drawbacks. First, reparameterizing the curve changes the density of samples along the curve. This density is now known to be a key aspect in CS $[2,6,7,19]$, since it directly impacts the number of required measurements to ensure exact recovery (noiseless case) or accurate (noisy case) reconstruction. Second, the challenge of rapid acquisitions is to reduce the scanning time (echo train duration) and limit geometric distortions and off-resonance effects by covering the $k$-space as fast as possible. The perfect fit to any arbitrary curve (support constraint) may be time consuming, especially in the high curvature parts of the trajectory. In particular, the time to traverse piecewise linear trajectories [2, 20-23] may become too long. Indeed, the magnetic field gradients have to be set to zero at each singular point of such trajectory. To overcome these two limitations, new gradient waveform design methods have to be pushed forward.

N. Chauffert and P. Ciuciu are with Inria-CEA Parietal team/ NeuroSpin center, CEA Saclay. Contact: philippe.ciuciu@cea.fr P. Weiss is with PRIMO Team, ITAV, USR 3505, Université de Toulouse.

J. Kahn is with Institut de Mathématiques de Toulouse, CNRS UMR 5219, Université de Toulouse. 


\section{A. Contributions}

In this paper, we propose an alternative to reparameterization based on a convex optimization formulation. Given any parameterized curve, our algorithm returns the closest curve that fulfills the gradient constraints. The main advantages of the proposed approach are the following: i) the time to traverse the $k$-space is fixed enabling to find the closest curve in a given time, ii) the distance between the input and output curves is the quantity to be minimized ensuring a low deviation to the original sampling distribution, iii) it is flexible enough to handle additional hardware constraints (e.g., trajectory starting from the $k$-space center, different kinematic constraints,...) in the same framework. We propose an efficient first order dual algorithm to solve the resulting problem and provide theoretical guarantees in terms of convergence rate. We also demonstrate through theory and numerical experiments that the distortion to the initial density is minimized compared to the reparameterization approach. We eventually illustrate the performance of our approach on simulations.

\section{B. Paper organization}

In Section II, we review the formulation of MRI acquisition, by recalling the gradient constraints and introducing the projection problem. Then, in Section III, it is shown that curves generated by the proposed strategy (initial parameterization followed by the projection onto the set of physical constraints) may be used to design MRI sampling schemes with locally variable densities. In Section IV, we provide an optimization algorithm to solve the projection problem, and estimate its rate of convergence. Next, the behavior of our algorithm is illustrated in Section V on two complementary cases: one popular sampling scheme, namely EPI trajectory and one VDS strategy (traveling salesman problem or TSP-based curve) ${ }^{1}$, yet advertising the usefulness of the proposed approach for practical MRI applications. The EPI readout allows us to illustrate the performance of our projection algorithm when sampling is performed at Shannon information rate whereas TSP-based trajectories are dedicated to compressed sensing illustrations. The pros and cons of our method are discussed in Section VI and concluding remarks are drawn in Section VII.

\section{DESIGN OF $k$-SPACE TRAJECTORIES USING PHYSICAL GRADIENT WAVEFORMS.}

In this section, we recall the standard modeling of the acquisition constraints in MRI [15, 17]. We justify the lack of accuracy of current reparameterization methods in the VDS context, and motivate the introduction of a new projection algorithm that preserves the sampling density.

\section{A. Sampling in MRI}

In MRI, images are sampled in the $k$-space domain along parameterized curves $s:[0, T] \mapsto \mathbb{R}^{d}$ where $d \in\{2,3\}$ denotes the image dimensions. The $i$-th coordinate of $s$ is denoted $s_{i}$. Let $u: \mathbb{R}^{d} \rightarrow \mathbb{C}$ denote a $d$ dimensional image and $\hat{u}$ be its Fourier transform. Given an image $u$, a curve $s:[0, T] \rightarrow \mathbb{R}^{d}$ and a sampling step $\Delta t$, the image $u$ shall be reconstructed using the set $^{2}$ :

$$
\mathcal{E}(u, s)=\left\{\hat{u}(s(j \Delta t)), 0 \leqslant j \leqslant\left\lfloor\frac{T}{\Delta t}\right\rfloor\right\} .
$$

\section{B. Gradient constraints}

The gradient waveform associated with a curve $s$ is defined by $g(t)=\gamma^{-1} \dot{s}(t)$, where $\gamma$ denotes the gyro-magnetic ratio [17]. The gradient waveforms being obtained by energizing gradient coils with electric currents, they are submitted to hardware constraints.

1) Kinematic constraints: Due to physical but also safety (ie avoid nerve stimulation) constraints, the electric currents passing through gradient coils have a bounded amplitude and cannot vary too rapidly (slew rate). Mathematically, these constraints read:

$$
\|g\| \leqslant G_{\max } \quad \text { and } \quad\|\dot{g}\| \leqslant S_{\max }
$$

where $\|\cdot\|$ denotes either the $\ell^{\infty}$-norm defined by $\|f\|_{\infty}:=\max _{1 \leq i \leq d} \sup _{t \in[0, T]}\left|f_{i}(t)\right|$, or the $\ell^{\infty, 2}$-norm defined by $\|f\|_{\infty, 2}:=$ $\sup _{t \in[0, T]}\left(\sum_{i=1}^{d}\left|f_{i}(t)\right|^{2}\right)^{\frac{1}{2}}$. These constraints might be Rotation Invariant (RIV) if $\|\cdot\|=\|\cdot\|_{\infty, 2}$ or Rotation Variant (RV) if $\|\cdot\|=\|\cdot\|_{\infty}$, depending on whether each gradient coil is energized independently from others or not. The set of kinematic constraints is denoted $\mathcal{S}$ :

$$
\mathcal{S}:=\left\{s \in\left(\mathcal{C}^{2}([0, T])\right)^{d},\|\dot{s}\| \leqslant \alpha,\|\ddot{s}\| \leqslant \beta\right\}
$$

where $\alpha=\gamma G_{\max }$ and $\beta=\gamma S_{\max }$.

\footnotetext{
${ }^{1}$ The reader interested in assessing the behavior of the proposed algorithm for other VDS trajectories like spiral and rosette may look at [24, Chap. 3].

${ }^{2}$ For ease of presentation, we assume that the values of $u$ in the $k$-space correspond to its Fourier transform and we neglect distortions occurring in MRI such as noise. We also neglect the energy decay due to signal relaxation.
} 
2) Additional affine constraints: Specific MRI acquisitions may require additional constraints, such as:

- Imposing that the trajectory starts from the $k$-space center (ie, $s(0)=0)$ to save time and avoid blips. The end-point can also be specified by $s(T)=s_{T}$.

- In the context of multi-shot MRI acquisition, several radio-frequency pulses are necessary to cover the whole $k$-space. Hence, it makes sense to enforce the trajectory to start from the $k$-space center at every $T R$ (repetition time) $)^{3}: s(m \cdot T R)=$ $0,0 \leqslant m \leqslant\left\lfloor\frac{T}{T R}\right\rfloor$.

- In addition to starting from the $k$-space center, one could impose the initial speed as for instance: $\dot{s}(0)=0$.

- To avoid artifacts due to flow motion in the object of interest, gradient moment nulling (GMN) techniques have been introduced in [25] for spin or gradient echo sequences. In terms of constraints, nulling out the $i^{\text {th }}$ moment reads $\int_{t=0}^{T E} t^{i} g(t) \mathrm{d} t=0$, where $T E$ denotes the echo time. For example, canceling out the first-order moment compensates the motion of spins moving with constant speed.

Each of these constraints can be modeled by an affine relationship. Hereafter, the set of affine constraints is denoted by $\mathcal{A}$ :

$$
\mathcal{A}:=\left\{s:[0, T] \rightarrow \mathbb{R}^{d}, A(s)=v\right\},
$$

where $v$ is a vector of parameters in $\mathbb{R}^{p}$ ( $p$ is the number of additional constraints) and $A$ is a linear mapping from the curves space to $\mathbb{R}^{p}$.

A sampling trajectory $s:[0, T] \rightarrow \mathbb{R}^{d}$ will be said to be admissible if it belongs to the set $\mathcal{S} \cap \mathcal{A}$. In what follows, we assume that this set is non-empty, ie $\mathcal{S} \cap \mathcal{A} \neq \emptyset$. Moreover, we assume, without loss of generality, that the linear constraints are independent (otherwise some could be removed).

\section{Finding an optimal reparameterization}

The traditional approach to design an admissible curve $s \in \mathcal{S}$ given an arbitrary curve $c:[0, T] \rightarrow \mathbb{R}^{d}$ consists of finding a reparameterization $r$ such that $s=c \circ r$ satisfies the physical constraints while minimizing the acquisition time. This problem can be cast as follows:

$$
T_{\text {Rep }}=\min T^{\prime} \text { such that } \exists r:\left[0, T^{\prime}\right] \mapsto[0, T], c \circ r \in \mathcal{S} \text {. }
$$

It can be solved efficiently using optimal control [15] or convex optimization [17]. The resulting solution $s=c \circ r$ has the same support as $c$. This method however suffers from an important drawback when used in the CS framework: it does not provide any control on the density of samples along the curve. For example, for a given curve support shown in Fig. 1(a), we illustrate the new parameterization (keeping the same support) and the corresponding magnetic field gradients (see Fig. 1(b) for a discretization of the curve and (c) for the gradient profile). We notice that the new parameterized curve has to stop at every angular point of the trajectory, increasing the time spent by the curve in the neighborhood of these points (and more points in the discretization of the curve in Fig. 1(b)). This phenomenon is likely to modify the sampling distribution, as illustrated in Section III.

The next part is dedicated to introducing an alternative method, by relaxing the constraint of keeping the same support as $c$.

\section{Projection onto the set of constraints}

Here, we propose to find the projection of the given input curve $c$ onto the set of admissible curves $\mathcal{S}$ :

$$
s^{*}:=\underset{s \in \mathcal{S} \cap \mathcal{A}}{\operatorname{argmin}} \frac{1}{2} d^{2}(s, c)=\underset{s \in \mathcal{S} \cap \mathcal{A}}{\operatorname{argmin}} \frac{1}{2}\|s-c\|_{2}^{2}
$$

where $d^{2}(s, c)=\|s-c\|_{2}^{2}:=\int_{t=0}^{T}\|s(t)-c(t)\|_{2}^{2} d t$. This method presents important differences compared to the above mentioned optimal control approach: i) the solution $s^{*}$ and $c$ usually have different supports (see Fig. 1(d)); ii) the sets composed of the discretization of $c$ and $s^{*}$ at a given sampling rate are close to each other (Fig. 1(e)); iii) the acquisition time $T$ is fixed and equal to that of the input curve $c$. The time to traverse a curve is generally different from optimal reparameterization. In particular for piecewise linear curves, it is generally lower (see Fig. 1(f) where $T<T_{\text {Rep }}$ ).

In the next section, we explain why the empirical distribution of the samples along the projected curve is closer to that of points lying on the input curve. Also, we illustrate how the parameterization can distort the sampling distribution.

\section{CONTROL OF THE SAMPLING DENSITY}

Recent works have emphasized the importance of the sampling density [2,6,7,19] in the CS-MRI framework, ie in an attempt to reduce the amount of acquired data while preserving image quality at the reconstruction step. The choice of an accurate sampling distribution is crucial since it directly impacts the number of required measurements. In this paper, we will denote by $\pi$ a distribution defined over the $k$-space $K$. The profile of this distribution can be obtained by theoretical 


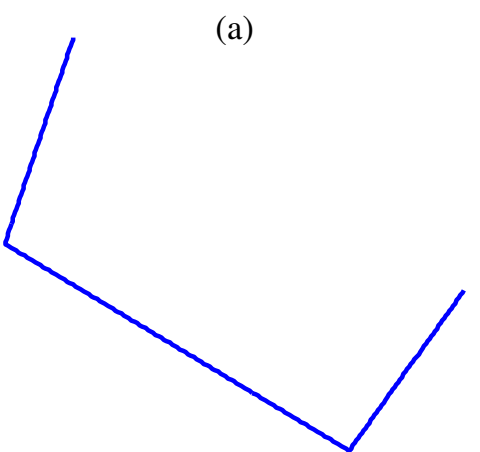

(d)

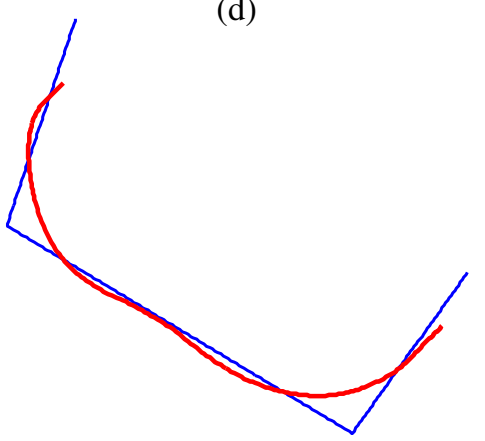

(b)

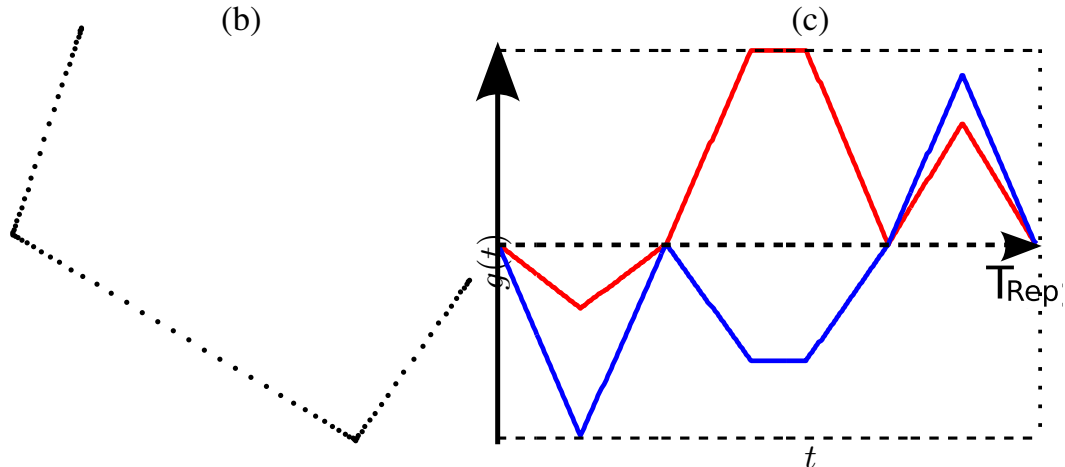

(e)

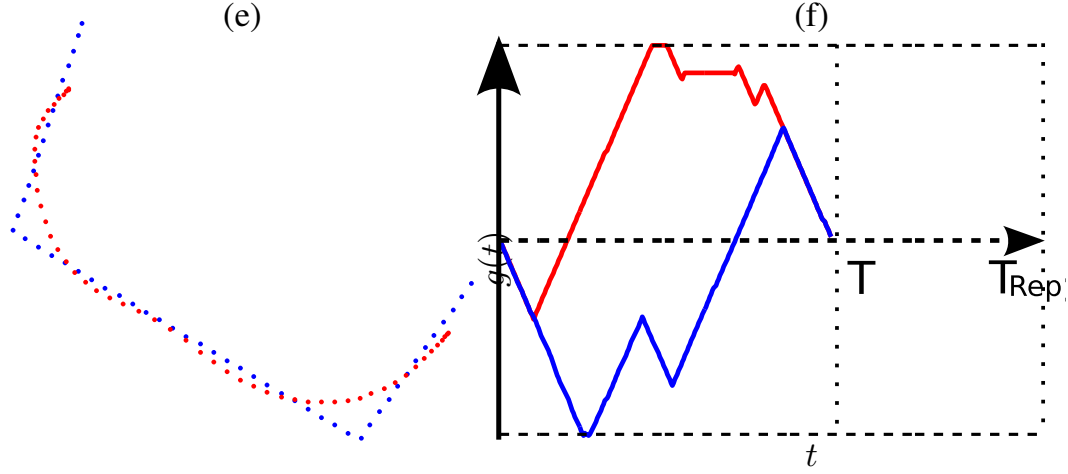

Fig. 1. Comparison of two methods to design gradient waveforms. Top row: Optimal control-based parameterization [15]. (a): input curve support. (b): discrete representation of the optimal reparameterization of the curve in $\mathcal{S}$. (c): corresponding gradient waveforms $\left(g_{x}, g_{y}\right)$. Dashed lines correspond to 0 and $+/-G_{\max }$. Bottom row: Illustration of the projection algorithm. (d): same input curve $c$ as in (a) parameterized at maximal speed, and the support of the projected curve $s^{*}$ onto $\mathcal{S}$. (e): discrete representation of the input and projected curves. (f): corresponding gradient waveforms $\left(g_{x}, g_{y}\right)$ with the same time scale as in (c): the time to traverse the $s^{*}$ is $39 \%$ shorter.

(a)

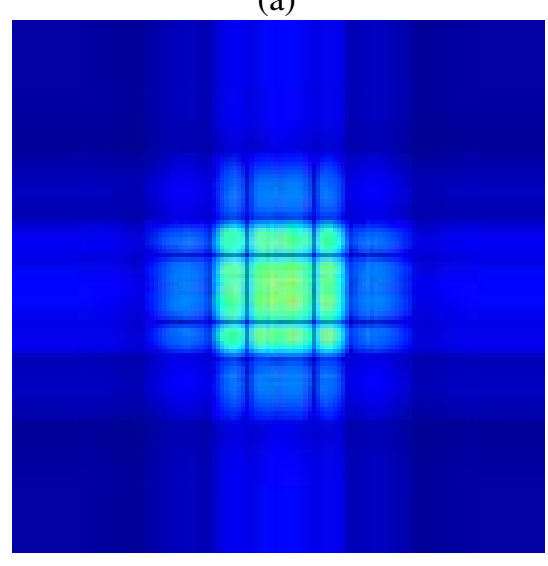

(b)

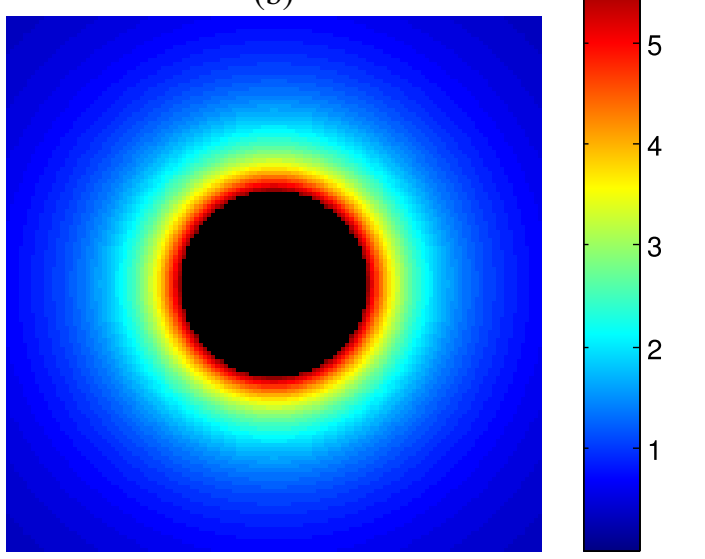

Fig. 2. Examples of 2D sampling distribution. (a): optimal distribution for a Symmlet transform [2, 6]. (b): radial distribution advocated in [2,7]: $p(k) \propto 1 /|k|^{2}$.

arguments $[2,6,7,19]$ leading to distributions as the one depicted in Fig. 2(a). Some heuristic distributions (e.g., radial) are known to perform well in CS-MRI experiments (Fig. 2(b)). A comparison between these two approaches can be found in [26].

The classical approach to design a trajectory that samples according to a fixed distribution while satisfying gradient constraints consists of:

1) Finding an input curve (admissible or not) $c$ with good distribution; We provide various strategies to achieve this step in Appendix A-B.

2) Estimating the fastest reparameterization of $c$ that belongs to the set of constraints.

In this paper, we suggest to replace the second step by:

$\left.2^{\prime}\right)$ Estimating $s^{*}$ the projection of $c$ onto the set of constraints, by solving problem (4).

${ }^{3}$ corresponding to the delivery of every radio-frequency pulse. 
We show that step 2') is preferable to step 2) since it better preserves the sampling density (or empirical measure). We begin by showing it through a theoretical study in paragraph III-A and then validate it through numerical experiments in paragraph III-B. The reader not interested by theoretical arguments can go directly to Subsection III-B.

\section{A. Theoretical study of the density control}

To formalize the notion of density, we need to introduce the definition of the empirical distribution of a curve.

Definition 1 (Empirical measure of a curve). Let $\lambda$ denote the Lebesgue measure and $\lambda_{T}=\frac{\lambda}{T}$ denote the Lebesgue measure normalized on the interval $[0, T]$. The empirical measure of a curve $s:[0, T] \mapsto K \subseteq \mathbb{R}^{d}$ is defined for any measurable set $\omega$ of $K$ as:

$$
P_{s}(\omega)=\lambda_{T}\left(s^{-1}(\omega)\right) \text {. }
$$

This definition means that the mass of a set $\omega$ is proportional to the time spent by the curve in $\omega$. To measure the distortion between an input curve and the projected one, we need to design a distance between measures. In this work, we propose to use the Wasserstein distance $W_{2}$ defined hereafter:

Definition 2 (Wasserstein distance $W_{2}$ ). Let $M$ be a domain of $\mathbb{R}^{d}$ and $\mathcal{P}(M)$ be the set of measures over $M$. For $\mu, \nu \in \mathcal{P}(M)$, $W_{2}$ is defined as:

$$
W_{2}(\mu, \nu)=\left(\inf _{\sigma \in \Pi(\mu, \nu)} \int\|x-y\|_{2}^{2} \mathrm{~d} \sigma(x, y)\right)^{\frac{1}{2}}
$$

where $\Pi \subset \mathcal{P}(M \times M)$ denote the set of measures over $M \times M$ with marginals $\mu$ and $\nu$ on the first and second factors, respectively.

$W_{2}$ is a distance over $\mathcal{P}(M)$ (see e.g., [27]). Intuitively, if $\mu$ and $\nu$ are seen as mountains, the distance is the minimum cost of moving the mountains of $\mu$ into the mountains of $\nu$, where the cost is the $\ell_{2}$-distance of transportation multiplied by the mass moved. Hence, the coupling $\sigma$ encodes the deformation map to turn one distribution $(\mu)$ into the other $(\nu)$.

Let us now analyze the distortion between the empirical distribution of the projected curve $P_{s^{*}}$ and the target distribution $\pi$. Since $W_{2}$ is a distance between measures, the triangle inequality holds:

$$
W_{2}\left(P_{s^{*}}, \pi\right) \leqslant \underbrace{W_{2}\left(P_{c}, \pi\right)}_{\text {Initial distortion }}+\underbrace{W_{2}\left(P_{s^{*}}, P_{c}\right)}_{\text {Projection distortion }} .
$$

The deviation is controlled by two terms: the initial distortion term $W_{2}\left(P_{c}, \pi\right)$ and the projection distortion term $W_{2}\left(P_{s^{*}}, P_{c}\right)$. The first term depends of the choice of the input curve $c$. This choice is crucial but is out the scope of this paper since it is not directly related to gradient waveform design. We still show in Appendix A that this term can be controlled precisely in the TSP case (for spiral imaging, again this is shown in [24, Chap. 3]).

We are now interested in controlling the Projection distortion term $W_{2}\left(P_{s^{*}}, P_{c}\right)$. The following proposition shows that the $W_{2}$ distance between the empirical distributions of the input and output curves ( $c$ and $s^{*}$, respectively) is controlled by the quantity $d\left(s^{*}, c\right)$ to be minimized when solving Eq. (4).

Proposition 1. For any two curves $s$ and $c:[0, T] \rightarrow \mathbb{R}^{d}$ :

$$
W_{2}\left(P_{s}, P_{c}\right) \leqslant d(s, c) .
$$

Proof: In terms of distributions, the quantity $d(s, c)$ reads:

$$
d^{2}(s, c)=\int_{M \times M}\|x-y\|_{2}^{2} \mathrm{~d} \sigma_{s, c}(x, y)
$$

where $\sigma_{s, c}$ is the coupling between the empirical measures $P_{s}$ and $P_{c}$ defined for all couples of measure sets $\left(\omega_{1}, \omega_{2}\right) \in M^{2}$ by $\sigma_{s, c}\left(\omega_{1}, \omega_{2}\right)=\frac{1}{T} \int_{t=0}^{T} 1_{\omega_{1}}(s(t)) 1_{\omega_{2}}(c(t)) \mathrm{d} t$, where $1_{\omega}$ denote the indicator function of $\omega$. The choice of this coupling is equivalent to choosing the transformation map as the association of locations of $c(t)$ and $s(t)$ for every $t$. We notice that the quantity to be minimized in Eq. (7) is an upper bound of $W_{2}\left(P_{s}, P_{c}\right)^{2}$, with the specific coupling $\sigma_{s, c}$.

To sum up, solving the projection problem (4) and finding $s^{*}$ amounts to minimizing an upper-bound of $W_{2}\left(P_{s^{*}}, \pi\right)$, the Wasserstein distance between the target density $\pi$ and the empirical distribution $P_{s^{*}}$, if we neglect the influence of the initial parameterization $c$. In some sense, our projection algorithm is therefore the best way to obtain a feasible curve and to preserve the input curve empirical measure. As will be seen in the next paragraph, densities are indeed much better preserved using projections than reparameterizations. 


\section{B. Numerical study of the density control}

Next, we performed simulations to show that the sampling density is better preserved using our algorithm compared to the optimal control approach. For doing so, we use traveling salesman-based (TSP) sampling trajectories [2,21], which are an original way to design random trajectories with a prescribed empirical distribution $\pi$ such as the one represented in Fig. 2(a). 10,000 such independent TSPs were drawn and parameterized with arc-length: note that these parameterizations are not admissible in general. Then, we sampled each trajectory at constant rate $\Delta t$ (as in Fig. 3 (top-row, left)), to form an histogram depicting the empirical distribution shown in Fig. 3 (top-row, center). The latter was eventually compared to $\pi$ in Fig. 3 (toprow, right). It is worth noting that the error was actually not close to zero, since the convergence result enounced in [2] is asymptotic, ie when the length of the TSP curve tends to infinity, whereas the latter remains bounded in this experiment.

In Fig. 3 (second row), we show that the classical reparameterization technique [15] leads to a major distortion of the sampling density, because of its behavior on the angular points already illustrated in Fig. 1(b). Then, we considered three constant speed parameterizations and projected them onto the same set of constraints $\left(G_{\max }=40 \mathrm{mT} . \mathrm{m}^{-1}\right.$ and $S_{\max }=150$ $\left.\mathrm{mT} \cdot \mathrm{m}^{-1} \cdot \mathrm{ms}^{-1}\right)$. Among these three initial candidates, we started by using an initial parameterization with low velocity $(10 \%$ of the maximal speed $\gamma G_{\max }$ with $\gamma=42.576 \mathrm{MHz}^{-1}$ for proton imaging). Its projection fits the sampling density quite well. Then, we increased the velocity to progressively reach $50 \%$ and even $100 \%$ of the maximal speed. The distortion of the sampling density of the projected curve increased, but remained negligible in contrast to what we observed for the exact reparameterization. Hence, this example illustrates that starting from a continuous trajectory with an empirical sampling distribution close to the target $\pi$, our projection algorithm yields feasible gradient waveforms while sampling the $k$-space along a discretized trajectory with empirical measure close to $\pi$ too.

\section{FINDING FEASIBLE WAVEFORMS USING CONVEX OPTIMIZATION}

Since the set of constraints $\mathcal{S} \cap \mathcal{A}$ is convex, closed and non-empty, Problem (4) always admits a unique solution. Even though $\mathcal{S}$ has a rather simple structure ${ }^{4}$, it is unlikely that an explicit solution to Problem (4) can be found. In what follows, we thus propose a numerical algorithm to compute the projection.

Problem discretization: A discrete-time curve $s$ is defined as a vector in $\mathbb{R}^{n \cdot d}$ where $n$ is the number of time points. Let $s(i) \in \mathbb{R}^{d}$ denote the curve location at time $(i-1) \delta t$ with $\delta t=\frac{T}{n-1}$. The discrete-time derivative $\dot{s} \in \mathbb{R}^{n \cdot d}$ is defined using first-order differences:

$$
\dot{\boldsymbol{s}}(i)= \begin{cases}0 & \text { if } i=1, \\ (\boldsymbol{s}(i)-\boldsymbol{s}(i-1)) / \delta t & \text { if } i \in\{2, \ldots, n\} .\end{cases}
$$

In the discrete setting, the first-order differential operator can be represented by a matrix $\dot{\mathrm{M}} \in \mathbb{R}^{n \cdot d \times n \cdot d}$, ie $\dot{s}=\dot{\mathrm{M} s}$. We define the discrete second-order differential operator by $\ddot{\mathbf{M}}=-\dot{\mathbf{M}}^{*} \dot{\mathbf{M}} \in \mathbb{R}^{n \cdot d \times n \cdot d}$.

An efficient projection algorithm: The discrete primal problem we consider is the same as (4) except that all objects are discretized. It reads:

$$
\min _{\boldsymbol{s} \in \mathcal{S} \cap \mathcal{A}} \frac{1}{2}\|\boldsymbol{s}-\boldsymbol{c}\|_{2}^{2},
$$

where $\mathcal{S}:=\left\{s \in \mathbb{R}^{n \cdot d},\|\dot{\mathrm{M}} s\| \leqslant \alpha,\|\ddot{\mathrm{M}} s\| \leqslant \beta\right\}$ with all norms discretized, and where $\mathcal{A}$ is the discretized version of $\mathcal{A}$. Next, the main idea is to take advantage of the structure of the dual problem of $\mathcal{P}$ to design an efficient projection algorithm. The following proposition specifies this dual problem and the primal-dual relationships.

Proposition 2. Let $\|\boldsymbol{q}\|_{*}:=\sup \langle\boldsymbol{s}, \boldsymbol{q}\rangle$ denote the dual norm of $\|\cdot\|$. The following equality holds:

$$
\min _{\boldsymbol{s} \in \mathcal{S} \cap \mathcal{A}} \frac{1}{2}\|\boldsymbol{s}-\boldsymbol{c}\|_{2}^{2}=\sup _{\boldsymbol{q}_{1}, \boldsymbol{q}_{2} \in \mathbb{R}^{n \cdot d}} F\left(\boldsymbol{q}_{1}, \boldsymbol{q}_{2}\right)-\alpha\left\|\boldsymbol{q}_{1}\right\|_{*}-\beta\left\|\boldsymbol{q}_{2}\right\|_{*},
$$

where

$$
F\left(\boldsymbol{q}_{1}, \boldsymbol{q}_{2}\right)=\min _{\boldsymbol{s} \in \mathcal{A}}\left\langle\dot{\mathbf{M}} \boldsymbol{s}, q_{1}\right\rangle+\left\langle\ddot{\mathbf{M}} \boldsymbol{s}, q_{2}\right\rangle+\frac{1}{2}\|\boldsymbol{s}-\boldsymbol{c}\|_{2}^{2} .
$$

Moreover, let $\left(\boldsymbol{q}_{1}^{*}, \boldsymbol{q}_{2}^{*}\right)$ denote any minimizer of the dual problem (8), $\boldsymbol{s}^{*}$ denote the unique solution of the primal problem $(\mathcal{P})$ and $\boldsymbol{s}^{*}\left(\boldsymbol{q}_{1}^{*}, \boldsymbol{q}_{2}^{*}\right)$ denote the solution of the minimization problem (9). Then $s^{*}=s^{*}\left(\boldsymbol{q}_{1}^{*}, \boldsymbol{q}_{2}^{*}\right)$.

Proof: The proof is given in Appendix B.

The following proposition gives an explicit expression of $s^{*}\left(\boldsymbol{q}_{1}^{*}, \boldsymbol{q}_{2}^{*}\right)$.

Proposition 3. The minimizer

$$
\boldsymbol{s}^{*}\left(\boldsymbol{q}_{1}^{*}, \boldsymbol{q}_{2}^{*}\right)=\underset{\boldsymbol{s} \in \mathcal{A}}{\arg \min }\left\langle\dot{\mathbf{M}} \boldsymbol{s}, \boldsymbol{q}_{1}\right\rangle+\left\langle\ddot{\mathbf{M}} \boldsymbol{s}, \boldsymbol{q}_{2}\right\rangle+\frac{1}{2}\|\boldsymbol{s}-\boldsymbol{c}\|_{2}^{2}
$$

${ }^{4}$ it is just a polytope when the $\ell^{\infty}$-norm is used. 
$\|P-\pi\|_{2} /\|\pi\|_{2}$
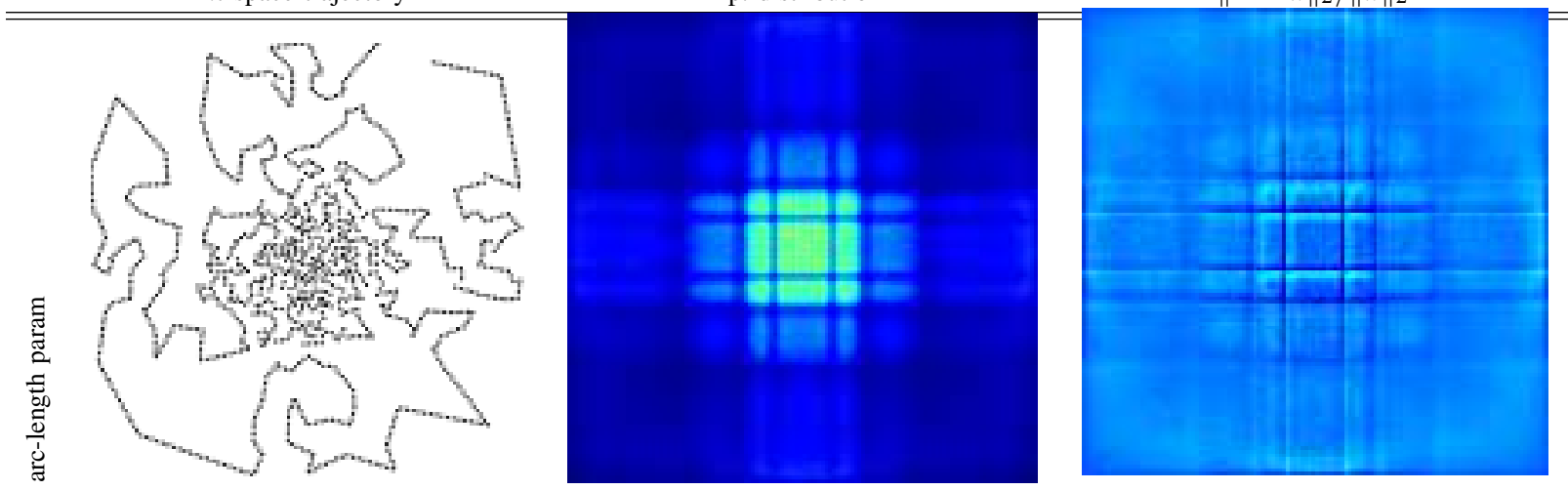

Not-admissible
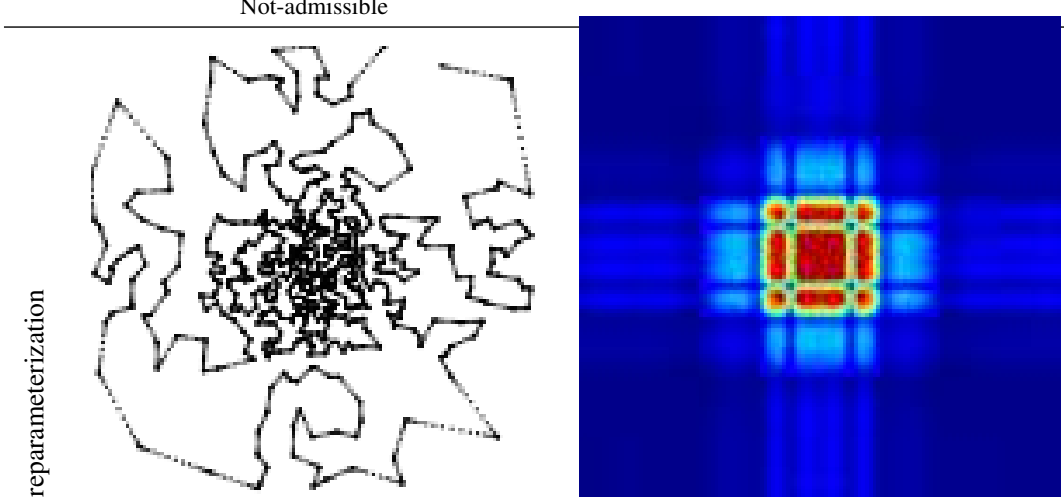

relative error $=12 \%$
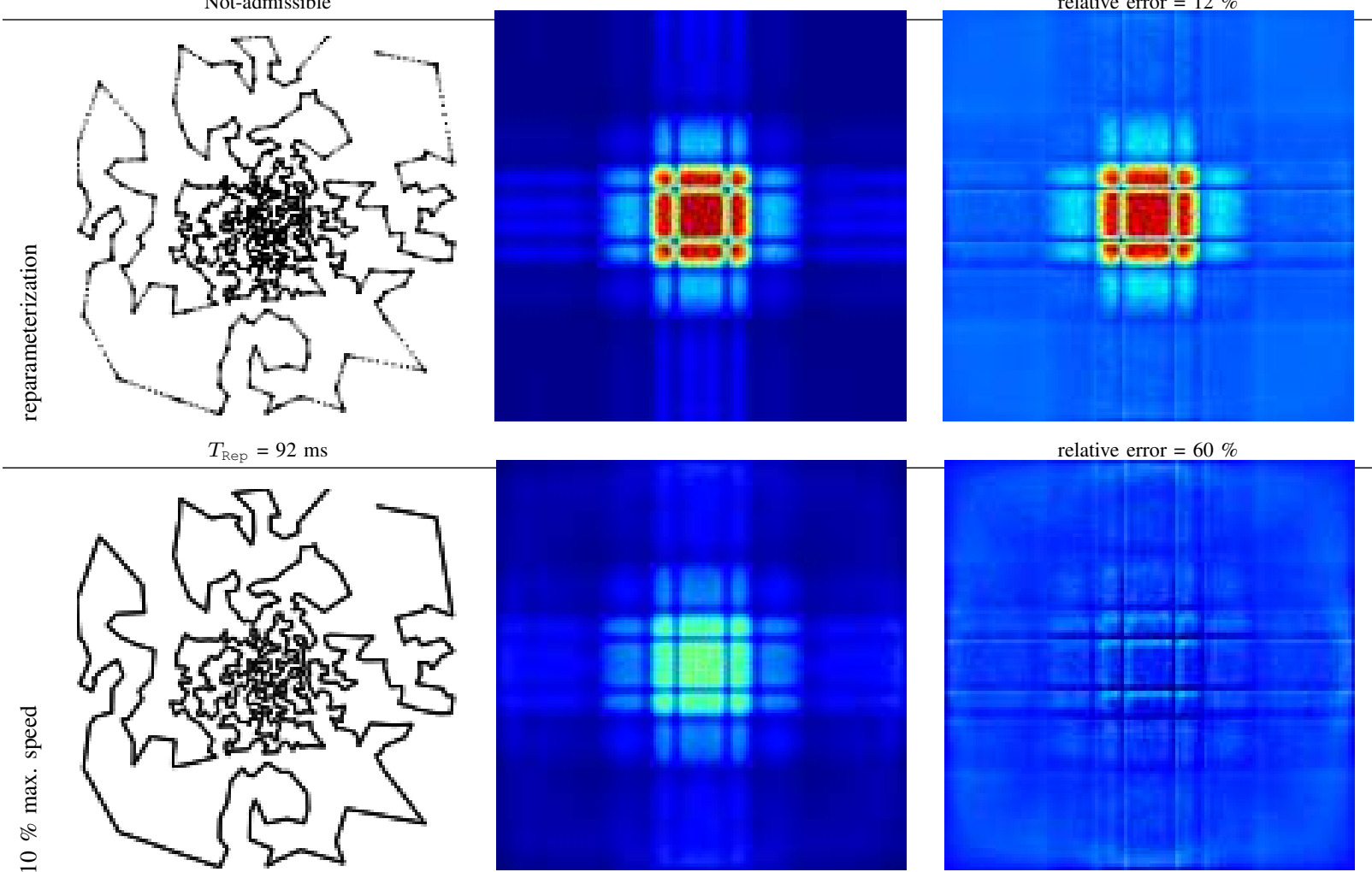

relative error $=60 \%$
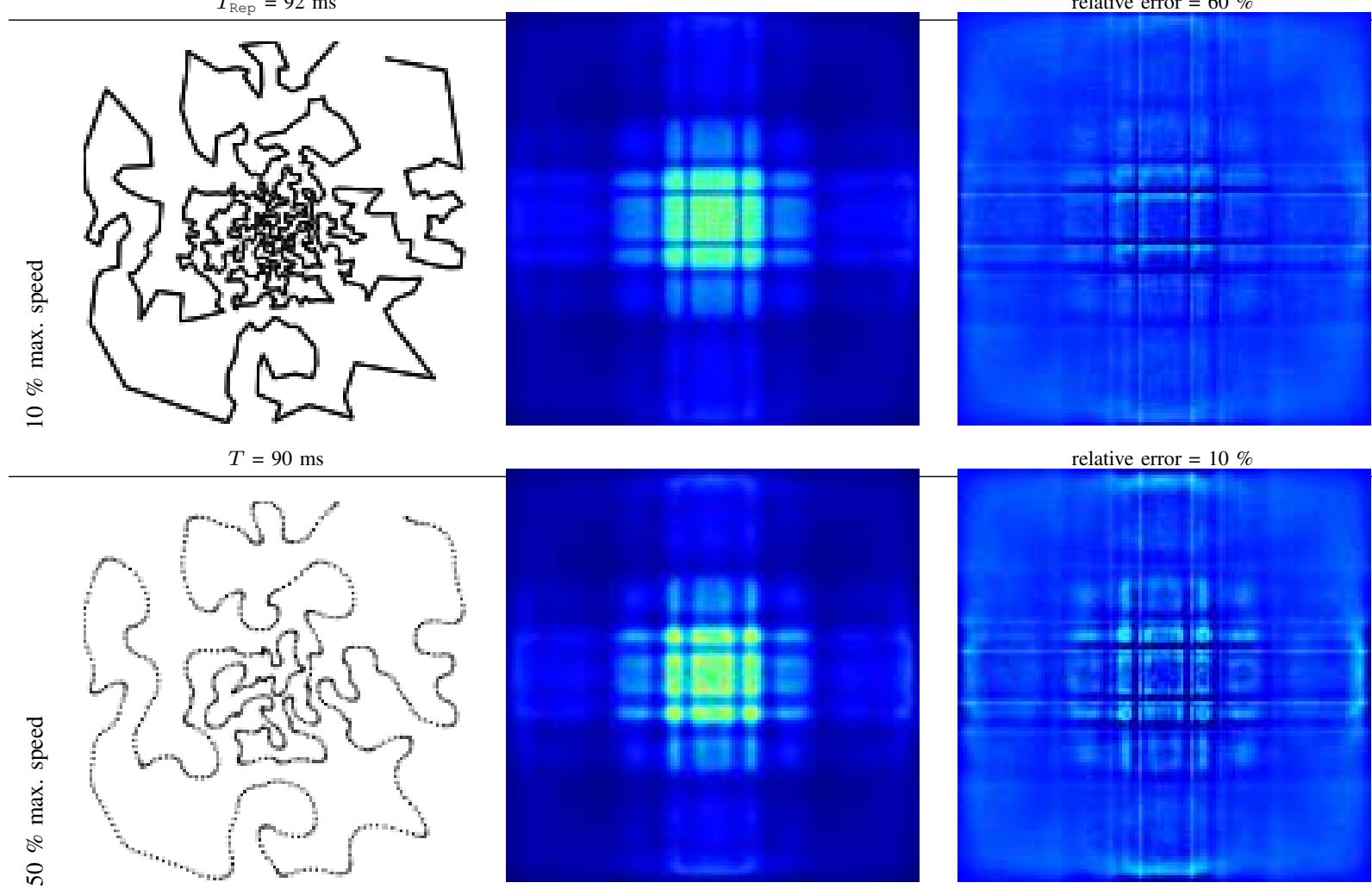

relative error $=10 \%$

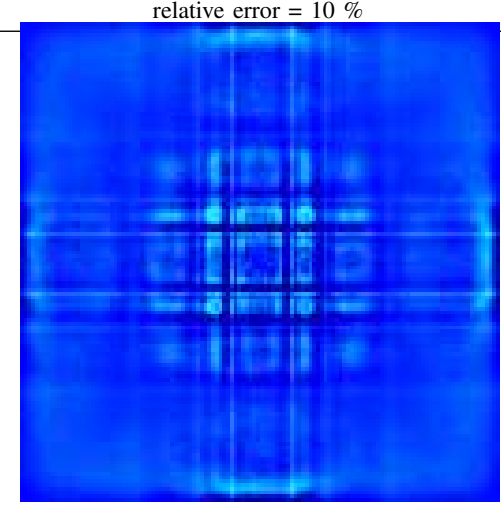

$T=18 \mathrm{~ms}$

relative error $=12 \%$
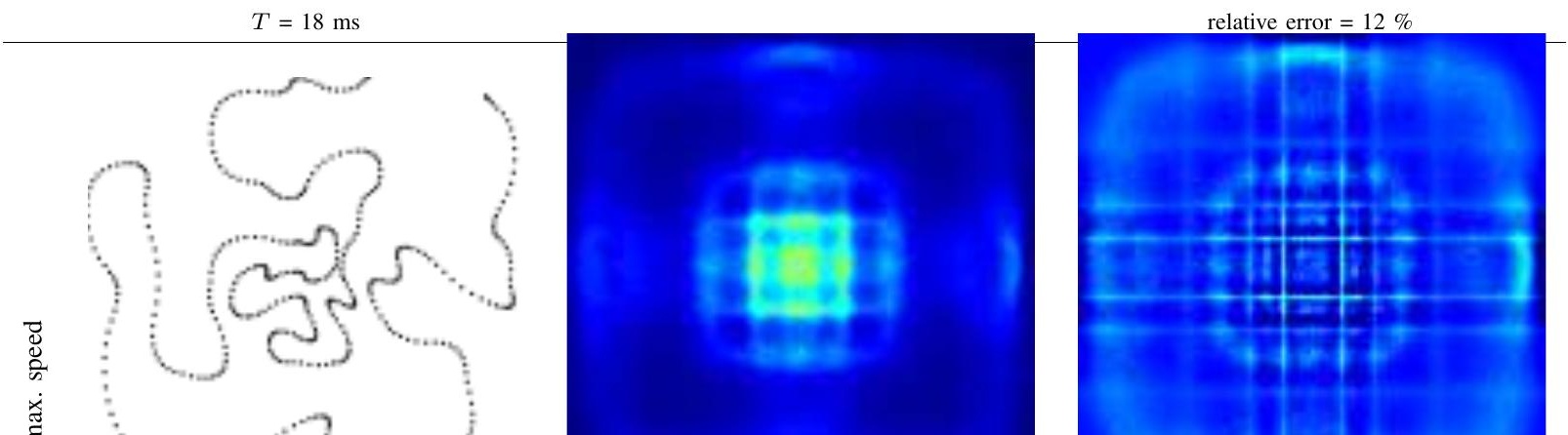
is given by

$$
\boldsymbol{s}^{*}\left(\boldsymbol{q}_{1}, \boldsymbol{q}_{2}\right)=\boldsymbol{z}+\boldsymbol{A}^{+}(\boldsymbol{v}-\boldsymbol{A} \boldsymbol{z})
$$

where $\boldsymbol{A} \in \mathbb{R}^{p \times n \cdot d}$ is a matrix encoding the affine constraints, and $\boldsymbol{A}^{+}=\boldsymbol{A}^{*}\left(\boldsymbol{A} \boldsymbol{A}^{*}\right)^{-1}$ denotes its pseudo-inverse ${ }^{5}$. In addition, $\boldsymbol{z}=\boldsymbol{c}-\dot{\mathbf{M}}^{*} \boldsymbol{q}_{1}-\ddot{\mathbf{M}}^{*} \boldsymbol{q}_{2}$.

Proof: The proof is given in Appendix C.

Let us now analyse the smoothness properties of $F$.

Proposition 4. Function $F\left(\boldsymbol{q}_{1}, \boldsymbol{q}_{2}\right)$ is concave differentiable with gradient given by

$$
\nabla F\left(\boldsymbol{q}_{1}, \boldsymbol{q}_{2}\right)=-\left(\begin{array}{l}
\dot{\mathbf{M}} \boldsymbol{s}^{*}\left(\boldsymbol{q}_{1}, \boldsymbol{q}_{2}\right) \\
\ddot{\mathbf{M}} \boldsymbol{s}^{*}\left(\boldsymbol{q}_{1}, \boldsymbol{q}_{2}\right)
\end{array}\right)
$$

Moreover, the gradient mapping $\nabla F$ is Lipschitz continuous with constant $L=||\left|\dot{\mathbf{M}}^{*} \dot{\mathbf{M}}+\ddot{\mathbf{M}} * \ddot{\mathbf{M}}\right|||$, where ||$|\mathbf{M}|||$ denotes the spectral norm of $\mathbf{M}$.

Proposition 4 is a direct application of [28, Theorem 1] (see also [29]). The dual problem (8) has a nice structure: it is the sum of a differentiable convex function $\tilde{F}\left(\boldsymbol{q}_{1}, \boldsymbol{q}_{2}\right)=-F\left(\boldsymbol{q}_{1}, \boldsymbol{q}_{2}\right)$ and of a simple convex function $G\left(\boldsymbol{q}_{1}, \boldsymbol{q}_{2}\right)=\alpha\left\|\boldsymbol{q}_{1}\right\|_{*}+\beta\left\|\boldsymbol{q}_{2}\right\|_{*}$. The sum $\tilde{F}+G$ can thus be minimized efficiently using accelerated proximal gradient descents [30] (see Algorithm 1 below).

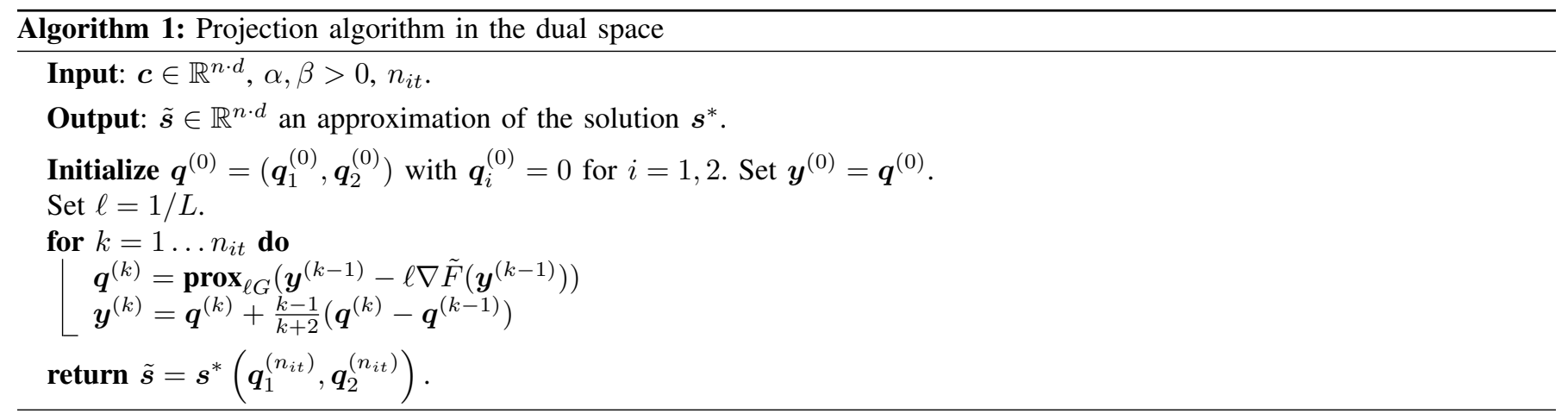

Moreover, by combining the convergence rate results of [30,31] and some convex analysis (see Appendix D), we obtain the following convergence rate:

Theorem 1. Algorithm 1 ensures that the distance to the minimizer decreases as $\mathcal{O}\left(\frac{1}{k^{2}}\right)$ :

$$
\left\|\boldsymbol{s}^{(k)}-\boldsymbol{s}^{*}\right\|_{2}^{2} \leq \frac{2 L\left\|\boldsymbol{q}^{(0)}-\boldsymbol{q}^{*}\right\|_{2}^{2}}{k^{2}} .
$$

\section{NUMERICAL EXPERIMENTS}

To compare our results with [15], we used the same gradient constraints. In particular, the maximal gradient norm $G_{\max }$ was set to $40 \mathrm{mT} . \mathrm{m}^{-1}$, and the slew-rate $S_{\max }$ to $150 \mathrm{mT} \cdot \mathrm{m}^{-1} \cdot \mathrm{ms}^{-1}$. We assume that the constraints are Rotation Invariant (RIV). The image field of view (FOV) is assumed to be $20 \mathrm{~cm}$ and $K_{\max }=N /(2 \cdot F O V)$ where $N$ is the target spatial grid size for image reconstruction. The sampling rate was fixed to $\Delta t=5 \mu \mathrm{s}$. For the ease of trajectory representation, we limit ourselves to $2 \mathrm{D}$ sampling curves, although our algorithm encompasses the $3 \mathrm{D}$ setting.

The Matlab codes embedding the projection algorithm as well as the scripts to reproduce our results are available at http://chauffertn.free.fr/codes.html. Hereafter, the affine constraints we considered are $s(0)=0$ and $\dot{s}(0)=0$. The nuling moments are not taken into account. However, they have been implemented in the code so that every end-user can play with. Simulations were performed on a Linux Ubuntu (64 bits) workstation with an Intel Xeon(R) CPU E5-2630 v2 @2.60GHz processor and $64 \mathrm{~GB}$ of RAM. The computation times required to generate the projected trajectories range from 2 min. (EPI) to $4 \mathrm{~min}$. (multi-shot TSP trajectory with 20,000 points). In all our numerical experiments, we observed that 10,000 iterations of the projection algorithm were enough to provide a good approximate solution.

To measure the impact of the proposed projection algorithm and compare it with the optimal reparameterization, we also performed image reconstruction and computed image quality in terms of signal-to-noise-ratio (SNR), self-similarity index (SSIM) and its complex wavelet extension (CW-SSIM) [32] since the latter is optimally designed when images are reconstructed in the wavelet domain. To this end, we performed simulations by starting from a high-resolution $N \times N$ MRI phantom $(N=1024)$ depicted in Fig. 5. Next, we undersampled its Fourier transform by the two competing sampling strategies

${ }^{5}$ Since the constraints are supposed to be linearly independent, $\boldsymbol{A}^{+}$is well-defined. 
and analyzed image quality after non-Cartesian $\ell^{1}$ reconstruction. All investigations related to EPI and TSP-based trajectories are depicted in Fig. 5 and Figs. 8-10, respectively. Quantitative results, corresponding traversal times and SNR/SSIM/CW-SSIM metrics of reconstructed images are summarized in Tabs. I-II.

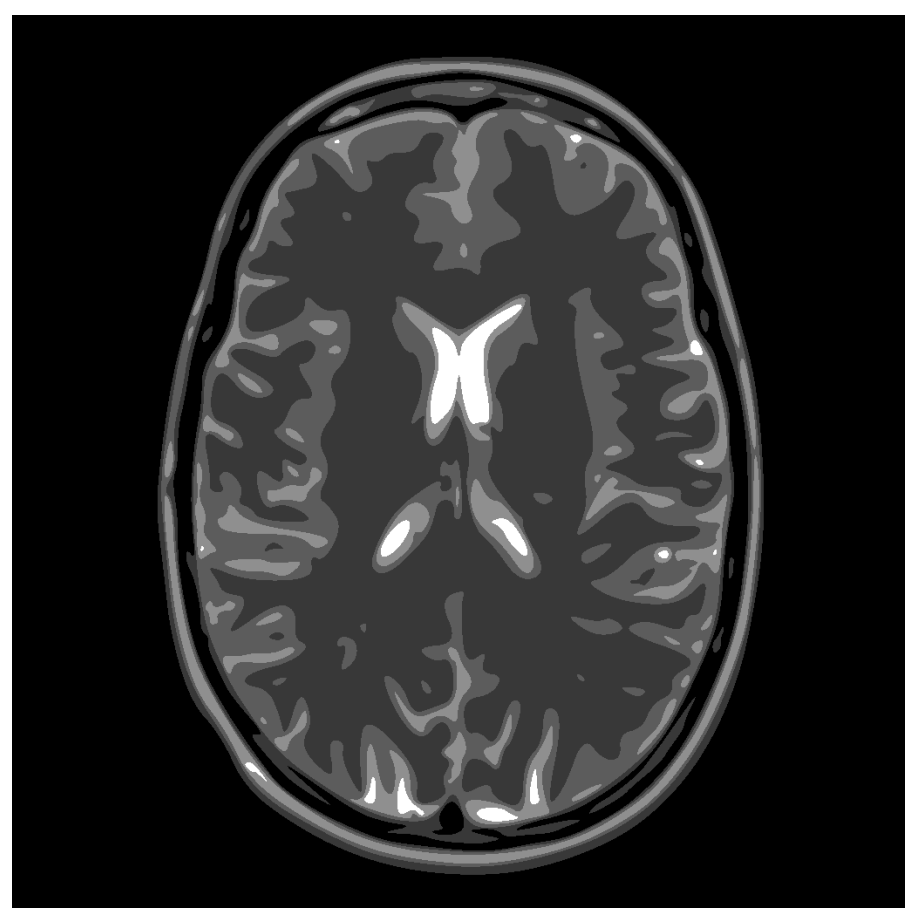

Fig. 4. MRI phantom of size $N \times N(N=1024)$ used for the experiments.

\section{A. Nonlinear image reconstruction}

We performed nonlinear image reconstruction as prescribed in the CS context [10,33]. Of importance, the samples locations $s(i), i=1, \ldots, n$ generated by the optimal reparameterization or our projection approach do not lie on a Cartesian grid. We therefore resorted to non-uniform Fourier transforms [34] to compute the $k$-space values. For comparison purposes, we started from a high resolution phantom $u$ (see Fig. 4 and [35]) that was used to compute the sets $\mathcal{E}\left(u, s_{\text {Rep }}\right)$ and $\mathcal{E}\left(u, s_{\text {proj }}\right)$. The latter are given by Eq. (1), where $s_{\text {Rep }}$ and $s_{\text {proj }}$ denote the optimal reparameterization and projected trajectory, respectively. Next, the images were reconstructed using $\ell_{1}$ regularization, ie:

$$
u^{*}=\underset{\tilde{u}}{\arg \min }\left\|\sum_{i=1}^{n}(\widehat{(u-\tilde{u}})(s(i))\right\|_{2}^{2}+\lambda\|\Phi \tilde{u}\|_{1}
$$

where $\Phi$ is a sparsifying transform (here Daubechies wavelets), $\lambda$ is a hyper-parameter ( $\lambda=10^{-4}$ was chosen for all numerical experiments), and $s$ is either $s_{\text {rep }}$ or $s_{\text {proj }}$. The minimizer of (13) was computed using accelerated proximal gradient descent [30, 36]. We actually checked that minimizing the penalized criterion (13) outperformed the solution of the basis pursuit (BP) problem by more than $1 \mathrm{~dB}[37,38]^{6}$ which rather computes the solution of $\ell_{1}$ minimization subject to the data consistency constraint. The reason lies in the fact that the NFFT computes fast summation approximations that may slightly violate the equality constraint involved in the BP formulation. The image solutions $\left(u_{\mathrm{Rep}}^{*}\right.$ and $\left.u_{\mathrm{proj}}^{*}\right)$ were then compared to a low resolution version of the $N \times N$ phantom where $N$ was either 128 or 256 , to compute image quality measures in Tabs. I-II.

On top of this, it is worth noting that we could still improve the SNR of reconstructed images by resorting either to more redundant decompositions such as tight frames [40] or even by learning dictionaries over which the image can be sparsely decomposed [41]. However, this aspect is beyond the scope of our current proof of concept.

\section{B. EPI trajectories}

EPI trajectories are a classical way of probing the $k$-space uniformly. In this section, we compared an EPI readout train with ramp-sampling (a sample was measured every $\Delta t$ from $t=0$ to $T_{\mathrm{Rep}}$ ) on $N=128$ lines, parameterized with optimal control and the projection approach that delivers a trajectory which traverses the $k$-space at constant speed.

\footnotetext{
${ }^{6}$ Implementation available in https://www.math.ucdavis.edu/ mpf/spgl1/ [38, 39].
} 
TABLE I

COMPARISON BETWEEN OPTIMAL REPARAMETERIZATION AND PROJECTION METHODS FOR AN EPI-LIKE TRAJECTORY AND $N=128$. THE BEST RESULTS IN TERMS OF READOUT TIME/IMAGE QUALITY TRADE-OFF ARE HIGHLIGHTED IN BOLD FONT.

\begin{tabular}{|c|cccc|}
\hline Method & $T(\mathrm{~ms})$ & SNR $(\mathrm{dB})$ & SSIM & CW-SSIM \\
\hline Optimal reparameterization 1 & 92 & 14.0 & 0.88 & 0.94 \\
Optimal reparameterization 2 & 110 & 43.7 & 0.99 & 1 \\
\hline Projection 1 & 83 & 21.8 & 0.97 & 0.97 \\
Projection 2 & $\mathbf{8 6}$ & $\mathbf{3 7 . 4}$ & $\mathbf{0 . 9 8}$ & $\mathbf{1}$ \\
\hline
\end{tabular}

The reparameterization approach requires setting the maximal speed $v_{\max }$. Two different values can be provided: the true maximal value $\alpha=\gamma G_{\max }$ where $G_{\max }$ is specified by the scanner manufacturer or the speed complying with Shannon sampling theorem $v=\Delta \xi / \Delta t$. The results using each setting are shown in Fig. 5, top-right and bottom-right respectively and the corresponding reconstructed images are depicted in Fig. 6(a)-(b). As can be seen, the first strategy yields a poor result with an SNR of $14.1 \mathrm{~dB}$ in $92 \mathrm{~ms}$, while the second one provides a good SNR of $43.7 \mathrm{~dB}$ in $110 \mathrm{~ms}$.

In order to apply our algorithm, we first need to design an input parameterization $c$. Two strategies were investigated. Based on Shannon sampling theorem, images will be accurately reconstructed if the distance between two consecutive $k$-space samples is below a threshold $\Delta \xi>0$. A good initial candidate $c$ consists of crossing the EPI trajectory at a constant speed $\|\dot{c}\|=v$ such that $v \Delta t=\Delta \xi$ (see Fig. 5, top-left). This condition will comply with Shannon theorem and thus ensure good reconstruction results. However, this initial guess is infeasible since the condition $\|\ddot{s}\| \leqslant \beta$ is violated on the extremities. We may thus project this initial trajectory on the admissible set $\mathcal{S}$. The result is shown on Fig. 5, top-center. The traversal time is $83 \mathrm{~ms}$ and the SNR of the image reconstructed with this sampling set is $21.8 \mathrm{~dB}$ (see all details in Tab. I and reconstruction in Fig. 6(c)). As can be seen, the projection shrinks the high frequencies along the $k_{x}$ axis. In order to avoid this pitfall, we can provide an initial parameterization that is slightly enlarged in the $k_{x}$ direction, see Fig. 5, bottom-left. This yields the result shown in Fig. 5, bottom-center, the traversal time grows up to $86.3 \mathrm{~ms}$ but the SNR of the reconstructed image also increases up to $37.4 \mathrm{~dB}$ (see Tab. I and reconstruction in Fig. 6(d)). From a perceptual point of view, the projection method yields a result that is visually indistinguishable from the optimal parameterization while being $28 \%$ faster.

Overall, this experiment highlights the two advantages of the proposed approach: i) the projection does not modify the initial sampling density and ii) it is able to smooth the corners, yielding faster acquisitions (from 10 to $28 \%$ ). Both ingredients lead to either a significant improvement in terms of image quality or a significant speed-up with no significant loss of image quality.
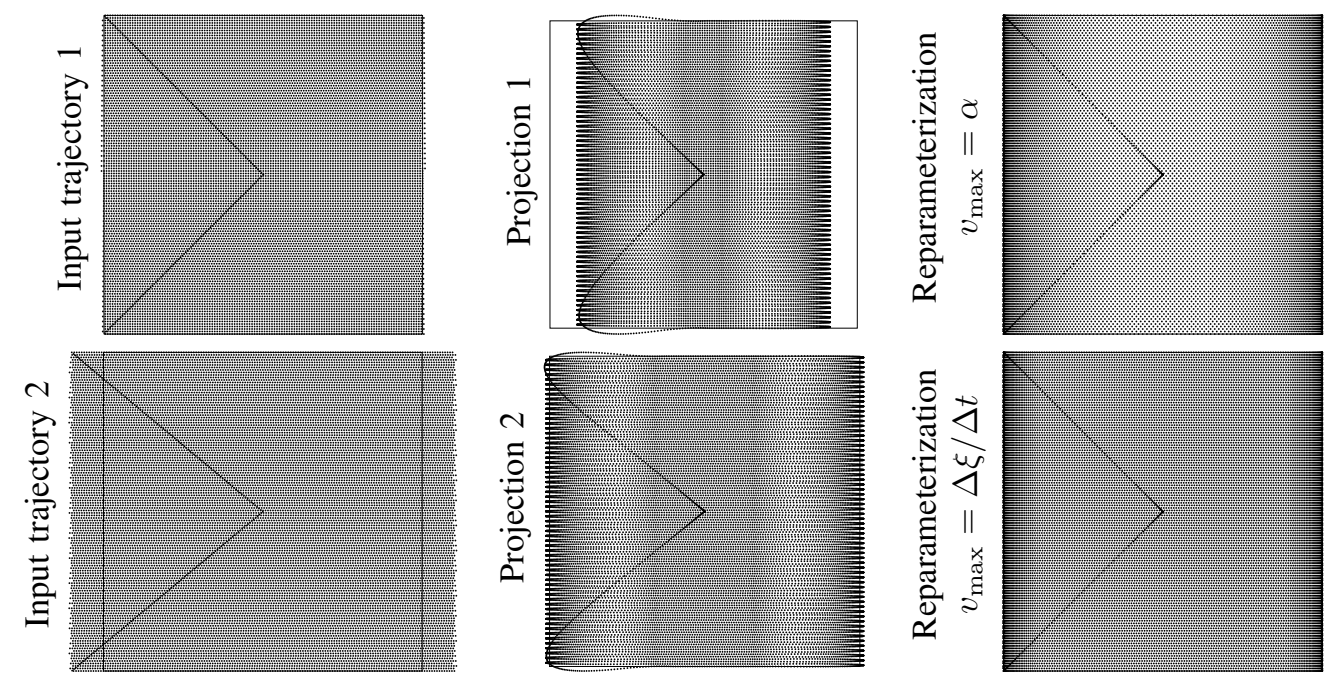

Fig. 5. Different strategies to parameterize an EPI trajectory based on optimal reparameterization or projection. The frame $\left[-K_{\max }, K_{\max }\right]^{2}$ is depicted with $K_{\max }=320 \mathrm{~m}^{-1}$. Horizontal and vertical axes stand for $k_{x}$ and $k_{y}$ directions, respectively.

\section{TSP sampling}

In the same spirit as Fig. 3, we performed numerical experiments using TSP trajectories in the single shot and multi-shot contexts [2,21]. Following our recent works [26,42], the drawing distribution of cities was chosen radial, with a decay of form $\min \left(c, 1 /(|k|+1)^{2}\right)$, where $c$ is a constant chosen so that the probability of drawing more than 2 samples per pixel is below $1 \%$. The sampling factor was defined as $100 \cdot N^{2} / n$, where $N^{2}$ is the number of pixels in the image and $n$ is the number of collected $k$-space samples.

We compared the reconstruction quality using reparameterization or the proposed projection approach, by generating trajectories with the same scanning time. To achieve this, we drew two sets of 3,000 and 20,000 "cities" (see Fig. 7-top) for 
(a): $\mathrm{SNR}=14 \mathrm{~dB}$

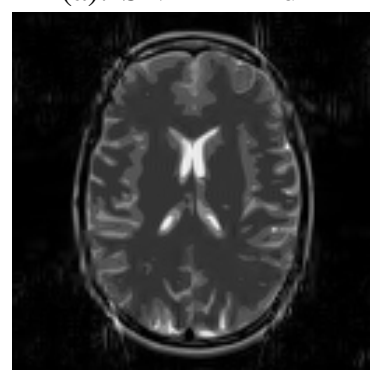

(c): $\mathrm{SNR}=21.8 \mathrm{~dB}$

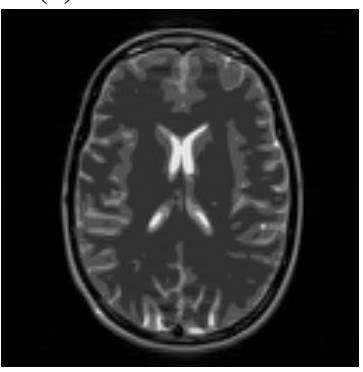

(b): $\mathrm{SNR}=43.7 \mathrm{~dB}$

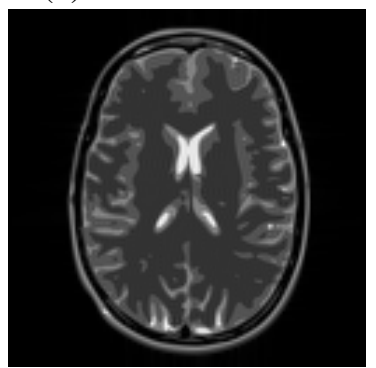

(d): SNR $=37.4 \mathrm{~dB}$

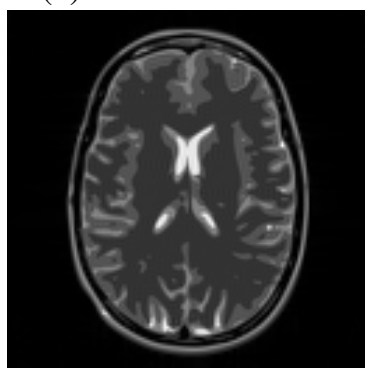

Fig. 6. Reconstructed images from data collected along EPI-like trajectories by solving (13). (a)-(b): Reconstruction results from the optimally reparameterized EPI readout (Top rows in Tab. I). (c)-(d): Reconstructed results from data collected using the projected EPI trajectories (Bottom rows in Tab. I).

TABLE II

COMPARISON BETWEEN OPTIMAL REPARAMETERIZATION AND PROJECTION METHODS ACCORDING TO SEVERAL CRITERIA FOR TSP-BASED TRAJECTORIES AND $N=256$. THE BEST RESULTS ARE HIGHLIGHTED IN BOLD FONT (NA: NOT APPLICABLE).

\begin{tabular}{|c|c|c|c|c|c|c|c|}
\hline Method & \# Shots & $T(\mathrm{~ms}) / \mathrm{shot}$ & Speed $v$ & Sampling Factor (\%) & SNR (dB) & SSIM & CW-SSIM \\
\hline \multirow{3}{*}{$\begin{array}{l}\text { Optimal reparameterization } \\
\qquad(3,000 \text { cities })\end{array}$} & 1 & 153 & NA & 58 & 11.9 & 0.68 & 0.86 \\
\hline & 2 & 79 & NA & 61 & 12.3 & 0.7 & 0.87 \\
\hline & 4 & 40 & NA & 61 & 12.8 & 0.72 & 0.88 \\
\hline \multirow{3}{*}{$\begin{array}{c}\text { Projection } \\
(20,000 \text { cities })\end{array}$} & 1 & 150 & $0.37 v_{\max }$ & 57 & 18.2 & 0.83 & 0.94 \\
\hline & 2 & 78 & $0.39 v_{\max }$ & 60 & 18.5 & 0.84 & 0.96 \\
\hline & 4 & 40 & $0.38 v_{\max }$ & 61 & 19 & 0.85 & 0.97 \\
\hline
\end{tabular}

standard resolution imaging $\left(N=256, K_{\max }=640 \mathrm{~m}^{-1}\right)$. The TSP solutions associated with the smaller and larger sets provide us with short and long trajectories, respectively. The shorter trajectory is traversed with optimal reparameterization in $T_{\mathrm{Rep}}=150 \mathrm{~ms}$, whereas the longer one can be parameterized at constant speed $v$ such that $T \simeq T_{\mathrm{Rep}}$. This parameterization is then used as an input trajectory to our projection algorithm (see Fig. 8).

The above mentioned traversal times are however too long to be insensitive to off-resonance effects and distortions during acquisition. Hence, to comply with reasonable readout times ( $40 \mathrm{~ms} \leqslant T \leqslant 80 \mathrm{~ms})$, hereafter we adopt a multi-shot acquisition strategy in which, as in the single shot case, the traversal times $T_{\text {Rep }}$ and $T$ associated with the reparameterization and projection algorithms have been matched ${ }^{7}$ to end up with meaningful comparisons. The reader may refer to Tab. II to get all information (traversal time, traversal speed for projection, sampling factor, SNR, SSIM, CW-SSIM) summarizing all our numerical experiments at this image resolution.

The multi-shot scenario relies on the same drawn "cities" as the single shot one. The $k$-space is segmented in $S$ slightly overlapping sub-domains (see Fig. 7 center and bottom rows) with $S \in\{2,4\}$. Afterwards, a TSP solver is run on each sub-domain (see Fig. 9-10, top rows). The interest for using slight overlap (20\%) between adjacent sub-domains lies in a good $k$-space sampling coverage around the boundaries. From these TSPs, the reparameterization and projection algorithms are run sequentially on each sub-domain. The outcomes of these algorithms are shown in Figs. 9-10, bottom rows. Importantly, as regards the projection algorithm, we have enabled the multi-shot constraint presented in Subsection II-B2 meaning that the first and last samples of each shot coincide with the $k$-space center.

We noticed that for a fixed traversal time $T$, the curve yielded by the projection algorithm provides a better $k$-space coverage as compared to optimal reparameterization. The main reason is that TSP trajectories embody singular points that require the gradients to be set to zero for each of them. Therefore, a sampling trajectory with singular points is time consuming. The main advantage of our algorithm is that the trajectory can be smoothed around these points, hence saving a lot of acquisition time.

At a fixed number of shots, the projection algorithm outperforms the optimal control approach by more than $6 \mathrm{~dB}$ in SNR

\footnotetext{
${ }^{7}$ For doing so, the speed $v$ of the initial parameterization for the projection method has been set by hand, see Tab. II for the corresponding values.
} 

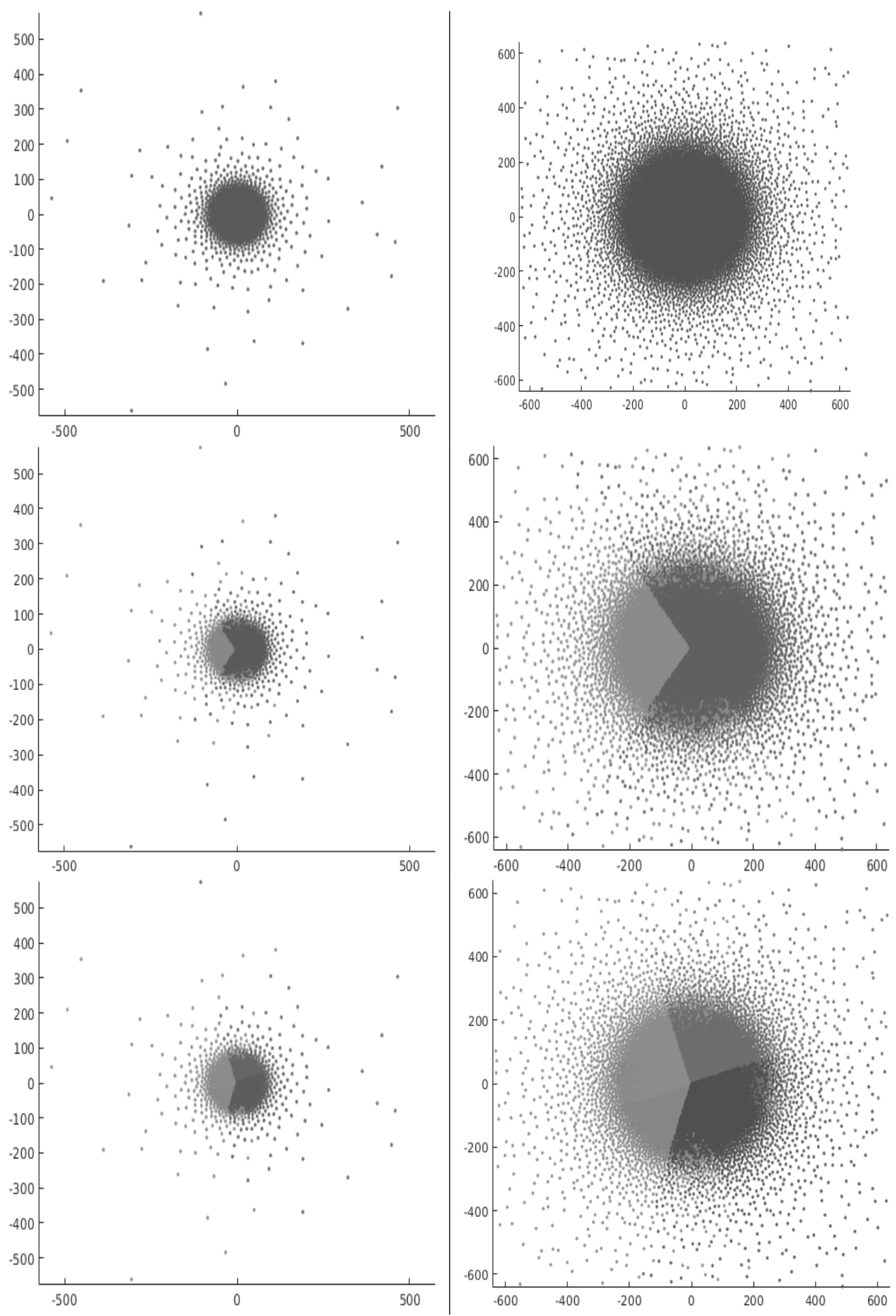

Fig. 7. The $k$-space has been decomposed into $S$ (top, center and bottom rows: $S=1, S=2$ and $S=4$, respectively) subdomains. When $S>1$, a slight overlap of $20 \%$ between adjacent subdomains (split into $2 \times 10 \%$ around each border) has been introduced so as to accurately sample the $k$-space part around the quadrant boundaries. In each subdomain, $N_{1} / S$ (left) and $N_{2} / S$ (right) cities (gray-scale coded) have been drawn where $N_{1}=3,000$ and $N_{2}=20,000$, respectively. 

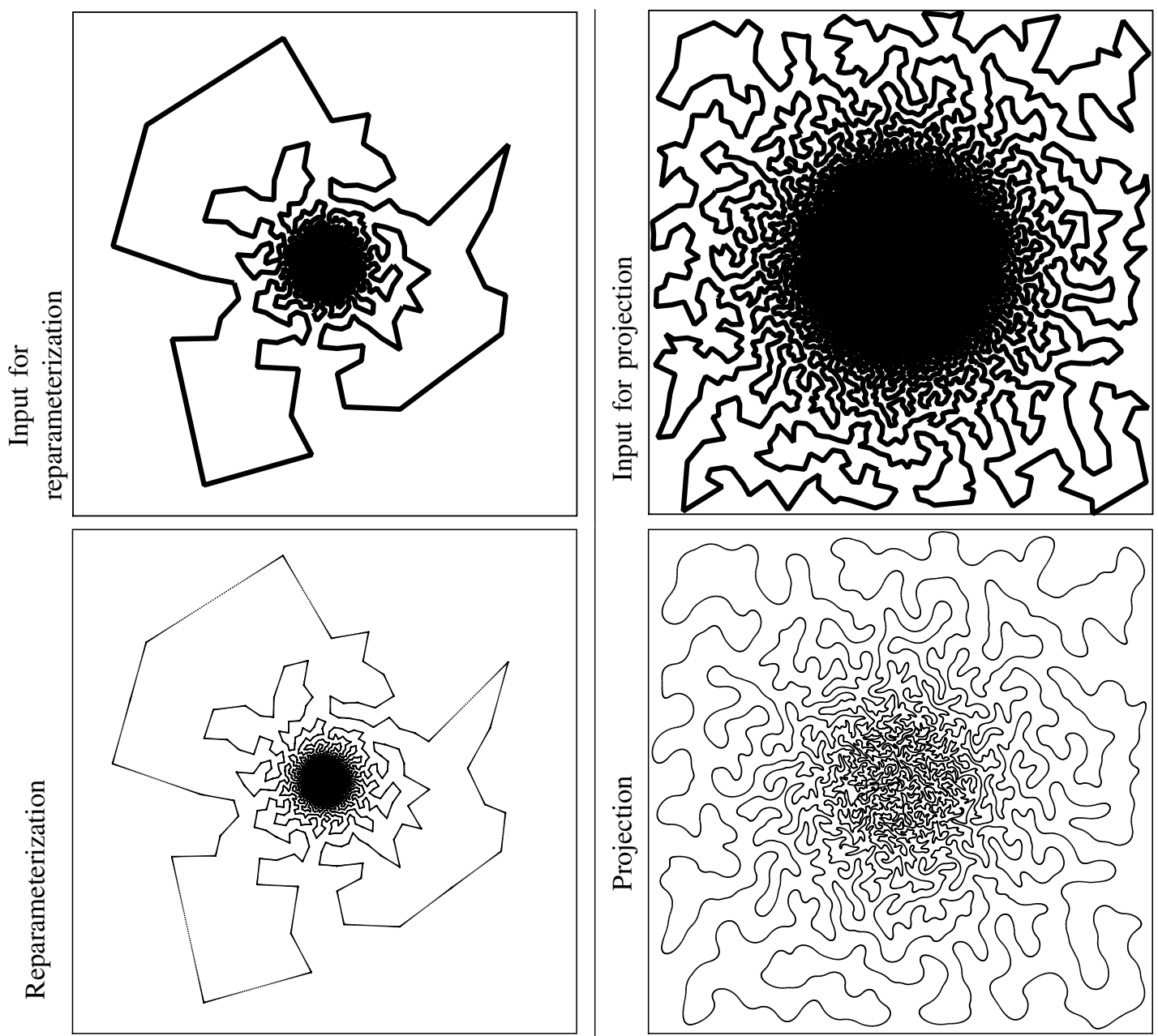

Fig. 8. Single-shot $(S=1)$ TSP-based trajectories for standard resolution imaging $\left(N=256, K_{\max }=640\right)$. Top row: Input curves to the reparameterization (left) and projection (right) algorithms generated as TSP solutions from $N_{1}=3,000$ (left) and $N_{2}=20,000$ (right) cities shown in Fig. 7-top using the Concorde solver. Bottom row: Output curves yielded by the optimal reparameterization and projection algorithms.

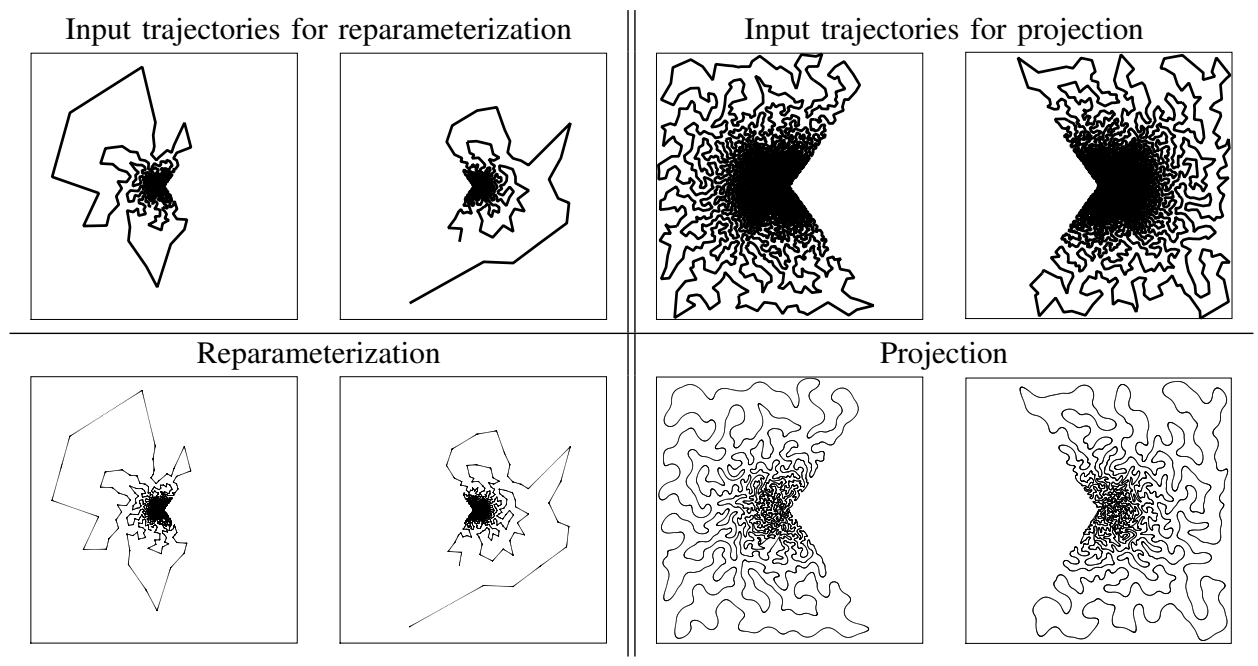

Fig. 9. 2-shot $(S=2)$ TSP-based trajectories with $20 \%$ of overlap between adjacent domains for standard resolution imaging $\left(N=256, K_{\max }=640\right)$. Top row: Input curves to the reparameterization (left) and projection (right) algorithms generated as TSP solutions from $N_{1} / S=1,500$ (left) and $N_{2} / S=$ 10, 000 (right) cities shown in Fig. 7-top using the Concorde solver. Bottom row: Output curves yielded by the optimal reparameterization and projection algorithms. Importantly, the multi-shot constraint which consists of passing through the $k$-space center at the beginning and end of each shot, has been enabled. 


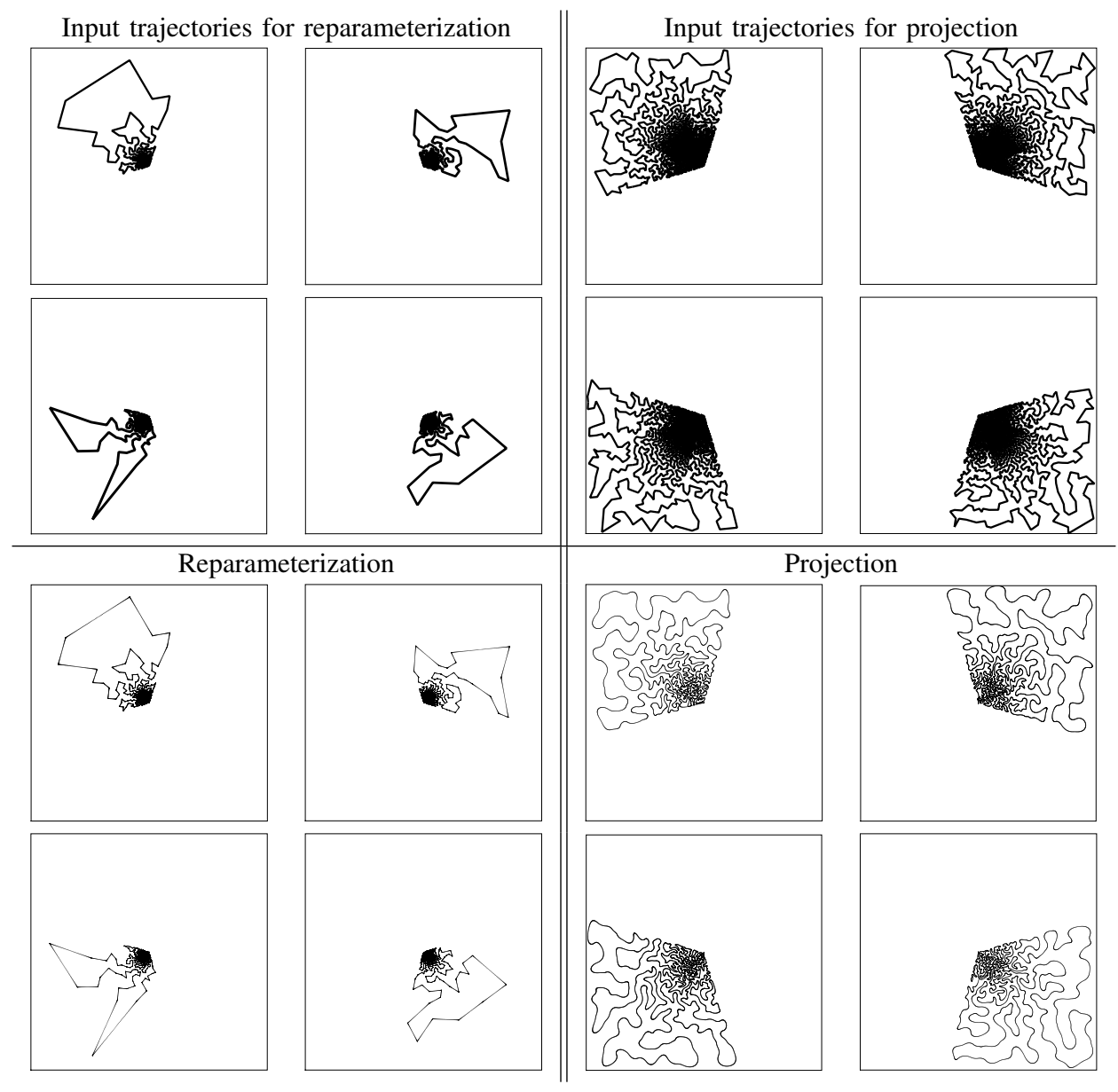

Fig. 10. 4-shot $(S=4)$ TSP-based trajectories with $20 \%$ of overlap between adjacent domains for standard resolution imaging $\left(N=256, K_{\max }=640\right)$. Top row: Input curves to the reparameterization (left) and projection (right) algorithms generated as TSP solutions from $N_{1} / S=750$ (left) and $N_{2} / S=$ 5, 000 (right) cities shown in Fig. 7-bottom using the Concorde solver. Bottom row: Output curves yielded by the optimal reparameterization and projection algorithms. Importantly, the multi-shot constraint which consists of passing through the $k$-space center at the beginning and end of each shot, has been enabled.

and 0.1 in SSIM/CW-SSIM indexes (see Tab. II and Fig. 11 for visual comparisons on reconstructed images). This result can be interpreted in the light of the significant difference of $k$-space coverage between the two approaches as illustrated in Figs. 9-10. For each method we also observed that increasing the number of shots slightly improves image quality since the sampling factor was slightly increased too (see Fig. 11). Indeed, we did not target a constant sampling rate across the different settings but instead the constraint $T \simeq T_{\text {Rep }}$ in each numerical experiment.

This set of simulations demonstrates that in given acquisition times and for TSP-based trajectories, our projection algorithm is a resolution enhancer (instead of a time saver) as compared to optimal reparameterization since it permits to explore wider $k$-spaces. The readout time for traversing the longer trajectory (20,000 cities) using optimal control would require $1.02 \mathrm{~s}$ in the single shot context and $(2 \times 530 \mathrm{~ms}, 4 \times 265 \mathrm{~ms})$ in $2 / 4$-shot scenarios (see supplementary materials), still for standard resolution $(N=256)$. In contrast, our method makes the readout of such trajectories feasible in the multi-shot setting $(4 \times 40 \mathrm{~ms})$, hence allowing a 6.6-fold acceleration in average. As a consequence, our projection algorithm could also be used as a time saver in this context.

\section{DISCUSSION}

In this paper, it has been shown that our projection algorithm has potential interests for smoothing sampling curves such as EPI or TSP-based trajectories. In this context, our algorithm delivers physically plausible trajectories while drastically reducing the traversal time and improving image quality. This is a direct consequence of its ability to project any piecewise linear initial parameterization onto admissible trajectories with different support. Finally, our projection algorithm can be mixed with other acceleration methods such as parallel imaging [43,44] or simultaneous multi-slice imaging technique [45].

Our projection method also provides a more accurate control of the sampling density. This has a positive impact on image reconstruction quality, as shown with the TSP experiments. Setting a fair trade-off between image quality and acquisition time is a usual concern in MRI that may depend on the application at hand (e.g., static vs dynamic imaging). Interestingly, our algorithm prescribes the acquisition time a priori. This provides the practitioner with an effective control on such trade-off. Hence, our 
(a): $\mathrm{SNR}=11.9 \mathrm{~dB}$

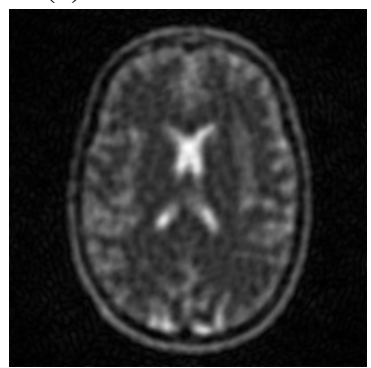

$(\mathrm{d}): \mathrm{SNR}=18.2 \mathrm{~dB}$

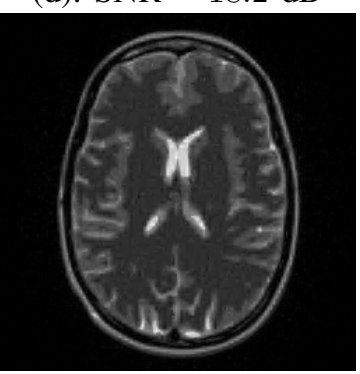

(b): $\mathrm{SNR}=12.3 \mathrm{~dB}$

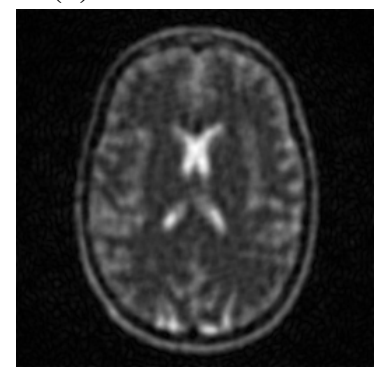

(e): SNR $=18.5 \mathrm{~dB}$

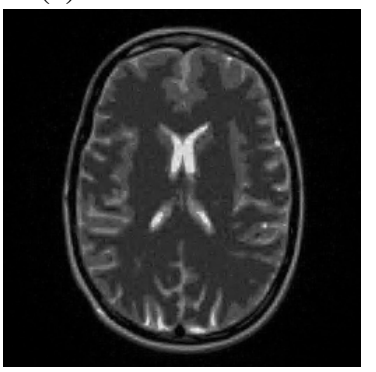

(c): $\mathrm{SNR}=12.8 \mathrm{~dB}$

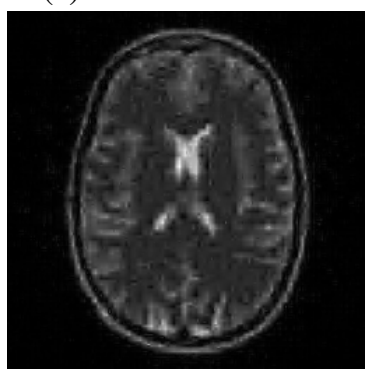

(f): $\mathrm{SNR}=19 \mathrm{~dB}$

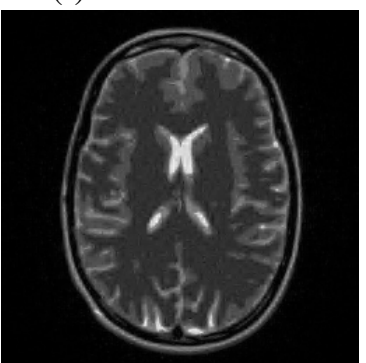

Fig. 11. Reconstructed images $(N=256)$ from data collected using the single (left) and multi-shots (2-shot in the center and 4-shot in the right) reparameterized (a)-(c) and projected (d)-(f) trajectories shown in Figs. 9- 10, respectively.

approach clearly compensates a major drawback of reparameterization methods that do not offer such control: the traversal time can be too fast hence an insufficient number of data are collected (EPI case), or too slow and not implementable (TSP-based sampling case).

On the other hand, our projection method has also limitations. In particular, the projected trajectory strongly depends on the initial parameterization. As we illustrated, parameterizing a given initial curve at different speeds provides very different projected trajectories. Also, we noticed in the context of spiral imaging that if the initial parameterization is not admissible (eg, angular speed too large), the output trajectory yielded by the projection algorithm concentrates on concentric circles corresponding to the maximal speed allowed by the gradient magnitude constraint. In the same context, the optimal reparameterization would not be impacted since it only depends on the support of the spiral. More generally, for smooth trajectories (like spiral and rosette), our projection algorithm will likely be outperformed by reparameterization approaches.

This clearly calls for extensions that might iterate until convergence between the two key steps, namely approximating the target density and finding an admissible trajectory from this approximation [46]. In such generalizations, the first step can be seen as a density-consistency stage where the sampled $k$-space locations might change from one iteration to the next to fit a target density. We believe that this idea might become the most important aspect of our contribution in the future: projections are one of the most basic tools from optimization and might serve in many different contexts. Preliminary results are available in [42].

As regards MRI applications, on the one hand the EPI readout is intensively used in diffusion-weigthed and functional MRI (fMRI). As such, any acceleration of this $k$-space trajectory or any EPI sophistication leading to improved image quality may have a significant impact, noticeably in neuroimaging. For instance, in high angular diffusion imaging [45] fast (or multiplexed) EPI may be used for fiber bundle tractography (structural connectivity) whereas in high temporal resolution fMRI, improved EPI may be useful for resting-state functional connectivity [47,48], for accurate estimation of the hemodynamic response function during evoked activity [49,50] or even for decoding brain activity [51]. On the other hand, the projection of TSP-based sampling onto the set of constraints has been successfully applied in a retrospective CS framework to MR angiography in the mouse brain [52]. Besides, an appealing application of our projection algorithm to TSP-based sampling is likely $T_{2}^{*}$ imaging. Indeed, the effective $T E_{\text {eff }}^{8}$ could be chosen around $30 \mathrm{~ms}$ by waiting for $10 \mathrm{~ms}$ after the radio-frequency pulse delivery and by splitting the readout ( $40 \mathrm{~ms}$ in the 4 -shot scenario) symmetrically around $T E_{\text {eff }}$. The sole constraint that has to be taken into account consists of starting the sampling from a location in the trajectory which is $10 \mathrm{~ms}$ far from the $k$-space center. Since the shots are actually designed as closing loops starting and ending at the center, this strategy is feasible in practice. Hence, the TSP-based multi-shot sampling might be applied to fMRI too since it allows us to shorten the echo train duration and to optimally select $T E_{\text {eff }}$ so as to maximize the blood oxygenated level-dependent contrast (e.g., $\mathrm{TE}_{\mathrm{eff}}=30 \mathrm{~ms}$ at $\left.3 \mathrm{~T}\right)$.

\footnotetext{
${ }^{8}$ The effective echo time is defined as the time to the echo which corresponds to the sampling of the $k$-space center (least phase encoding).
} 


\section{CONCLUSION}

We developed an algorithm to project any parameterized curve onto the set of curves which can be implemented on actual MRI scanners. Our method is an alternative to the existing gradient waveform design based on optimal control. The major advantages are that: i) the sampling time is fixed, which is crucial to adapt the proposed scheme to any MR imaging modality; ii) the sampling density is close to the target one, as required by compressed sensing theory; iii) the behavior of our algorithm provides shorter $k$-space coverage when the trajectory comprises numerous high curvature points, as illustrated in the TSP and EPI cases.

\section{ACKNOWLEDGMENTS}

This work benefited from the support of the FMJH Program Gaspard Monge in optimization and operation research, and from the support to this program from EDF. We are also grateful to our colleagues Dr Alexandre Vignaud and Dr Johanna Vannesjo who carefully reread the manuscript to better target the MRI readership, and Elvis Dohmatob for his remarks on convex optimization. Finally we would like to thank the three anonymous reviewers for their numerous comments which helped improving the paper.

\section{APPENDIX A}

DENSITY DEVIATION, CONTROL OF $W_{2}$-DisTANCE.

In Section III-A, we aim at controlling the Wasserstein distance $W_{2}\left(P_{s^{*}}, \pi\right)$, where $\pi$ is a target fixed sampling distribution, and $P_{s^{*}}$ is the empirical distribution of the projected curve. We used the triangle inequality (6) to bound this quantity by $W_{2}\left(P_{s^{*}}, P_{c}\right)+W_{2}\left(P_{c}, \pi\right)$. Here, we show that the quantity $W_{2}\left(P_{c}, \pi\right)$ can be as small as possible if $c$ is Variable Density Sampler (VDS) [2]. First, we define the concept of VDS, and then we provide an example based on TSP. The readers who might be interested in spiral VDS sampling may refer to [24, Chap. 3]. Next, we show that if $c$ is a VDS, $W_{2}\left(P_{c}, \pi\right)$ tends to 0 as the length of $c$ tends to infinity.

\section{A. Definition of a VDS}

First, we need to introduce the definition of weak convergence for measure:

Definition 3. A sequence of measures $\mu_{n} \in \mathcal{P}(K)$, the set of distributions defined over $K$, is said to weakly converge to $\mu$ if for any bounded continuous function $\phi$

$$
\int_{K} \phi(x) \mathrm{d} \mu_{n}(x) \rightarrow \int_{K} \phi(x) \mathrm{d} \mu(x) .
$$

We use the notation $\mu_{n} \rightarrow \mu$.

According to [2], a (generalized) $\pi$-VDS is a set of times $T_{n}$, such that $T_{n} \rightarrow \infty$ when $n \rightarrow \infty$, and a sequence of curves $c_{T_{n}}:\left[0, T_{n}\right] \rightarrow \mathbb{R}^{d}$ such that $P_{c_{T_{n}}} \rightarrow \pi$ when $n$ tends to infinity. A consequence of the definition is that the relative time spent by the curve in a part of the $k$-space is proportional to its density. Before showing that this implies that $W_{2}\left(P_{c_{T_{n}}}, \pi\right)$ tends to 0 , we give an example of VDS.

\section{B. VDS example: TSP sampling}

Hereafter, we give a single example based on TSP sampling to design continuous sampling trajectories that match a given distribution. In this context, TSP sampling provides a sequence of curves, hence a sequence of empirical measures that weakly converge to the target density.

The idea of using the shortest path amongst a set of points (the "cities") to design continuous trajectories with variable densities has been justified in [2,20]. Let us draw $n k$-space locations uniformly according to a density $q$ define over the $d$-dimensional (noted $d \mathrm{D}$ in what follows) $k$-space $(d=2$ or 3 ), and join them by the shortest path (the Traveling Salesman solution). Then, denote by $c_{n}$ a constant-speed parameterization of this curve. Define the density:

$$
\pi=\frac{q^{(d-1) / d}}{\int q^{(d-1) / d}(x) \mathrm{d}(x)}
$$

Then $P_{c_{n}} \rightarrow \pi$ when the number of cities $n$ tends to infinity.

The Traveling salesman-based sampling strategy is efficient to cover the $k$-space according to a target distribution, as depicted in Fig. 8-top in the single shot context where the VDS is achieved for the distribution shown in Fig. 2-top. Of course, the TSP approach also applies to any 3D density. 


\section{Control of $W_{2}$ distance}

Let us now assume without loss of generality that $K=\left[-k_{\max }, k_{\max }\right]^{d}$.

Let us recall a central result about $W_{2}$ (see e.g.,[27]):

Proposition 5. Let $M \subset \mathbb{R}^{d}, \mu \in \mathcal{P}(M)$ and $\mu_{n}$ be a sequence of $\mathcal{P}(M)$. Then, if $M$ is compact

$$
\mu_{n} \rightarrow \mu \Leftrightarrow W_{2}\left(\mu_{n}, \mu\right) \rightarrow 0
$$

An immediate consequence of this proposition and of the compactness of $K$ is the following proposition:

Proposition 6. Let $\left(c_{T_{n}}\right)_{n \geqslant 1}$ be a $\pi$-VDS, and $\varepsilon>0$. Then, there exists $n \geqslant 1$ such that $c_{T_{n}}:\left[0, T_{n}\right] \rightarrow K$ fulfills:

$$
W_{2}\left(P_{c_{T_{n}}}, \pi\right) \leqslant \varepsilon \text {. }
$$

To sum up, Proposition 6 ensures that we can find an input curve which empirical distribution is as close to the target distribution $\pi$ as we want.

\section{APPENDIX B}

ProOF OF PROPOSITION 2

Definition 4 (indicator function). Let $\boldsymbol{B} \subseteq \mathbb{R}^{n}$. The indicator of $\boldsymbol{B}$ is denoted $\imath_{\boldsymbol{B}}$ and defined by:

$$
\imath_{\boldsymbol{B}}(x)= \begin{cases}0 & \text { if } \boldsymbol{x} \in \boldsymbol{B} \\ +\infty & \text { otherwise }\end{cases}
$$

Let us now recall a classical result of convex optimization [29, P. 195]:

Proposition 7. Let $\boldsymbol{B}_{\alpha}=\left\{\boldsymbol{x} \in \mathbb{R}^{n},\|\boldsymbol{x}\| \leqslant \alpha\right\}$. Then the following identity holds:

$$
\imath_{\boldsymbol{B}_{\alpha}}(\boldsymbol{x})=\sup _{\boldsymbol{y} \in \mathbb{R}^{n}}\langle\boldsymbol{x}, \boldsymbol{y}\rangle-\alpha\|\boldsymbol{y}\|_{*} .
$$

Now, we can prove Proposition 2.

$$
\begin{aligned}
& \min _{\boldsymbol{s} \in \mathcal{S} \cap \mathcal{A}} \frac{1}{2}\|\boldsymbol{s}-\boldsymbol{c}\|_{2}^{2} \\
& =\min _{\boldsymbol{s} \in \mathcal{A}} \frac{1}{2}\|\boldsymbol{s}-\boldsymbol{c}\|_{2}^{2}+\imath_{\boldsymbol{B}_{\alpha}}(\dot{\mathbf{M}} \boldsymbol{s})+\imath_{\boldsymbol{B}_{\beta}}(\ddot{\mathbf{M}} \boldsymbol{s}) \\
& =\min _{\boldsymbol{s} \in \mathcal{A}} \frac{1}{2}\|\boldsymbol{s}-\boldsymbol{c}\|_{2}^{2}+\sup _{\boldsymbol{q}_{1}, \boldsymbol{q}_{2} \in \mathbb{R}^{n \cdot d}}\left\langle\dot{\mathbf{M}} \boldsymbol{s}, \boldsymbol{q}_{1}\right\rangle-\alpha\left\|\boldsymbol{q}_{1}\right\|_{*}+\left\langle\ddot{\mathbf{M}} \boldsymbol{s}, \boldsymbol{q}_{2}\right\rangle-\beta\left\|\boldsymbol{q}_{2}\right\|_{*} \\
& =\sup _{\boldsymbol{q}_{1}, \boldsymbol{q}_{\boldsymbol{q}} \in \mathbb{R}^{n \cdot d}} \min _{\boldsymbol{s} \in \mathcal{A}} \frac{1}{2}\|\boldsymbol{s}-\boldsymbol{c}\|_{2}^{2}+\left\langle\boldsymbol{s}, \dot{\mathbf{M}}^{*} \boldsymbol{q}_{1}\right\rangle+\left\langle\boldsymbol{s}, \ddot{\mathbf{M}}^{*} \boldsymbol{q}_{2}\right\rangle-\alpha\left\|\boldsymbol{q}_{1}\right\|_{*}-\beta\left\|\boldsymbol{q}_{2}\right\|_{*}
\end{aligned}
$$

The relationship between the primal and dual solutions reads $\boldsymbol{s}^{*}=\underset{\boldsymbol{s} \in \mathcal{A}}{\arg \min } \frac{1}{2}\|\boldsymbol{s}-\boldsymbol{c}\|_{2}^{2}+\left\langle\boldsymbol{s}, \dot{\mathbf{M}}^{*} \boldsymbol{q}_{1}^{*}\right\rangle+\left\langle\boldsymbol{s}, \ddot{\mathbf{M}}^{*} \boldsymbol{q}_{2}^{*}\right\rangle$. The sup and the min can be swapped at the third line, due to standard theorems in convex analysis (see e.g. [53, Theorem 31.3]).

\section{APPENDIX C}

Proof OF PROPOSITIONS 3

To show Proposition 3, first remark that

$$
\begin{aligned}
& \underset{\boldsymbol{s} \in \mathcal{A}}{\arg \min }\left\langle\dot{\mathbf{M}} \boldsymbol{s}, \boldsymbol{q}_{1}\right\rangle+\left\langle\ddot{\mathbf{M}} \boldsymbol{s}, \boldsymbol{q}_{2}\right\rangle+\frac{1}{2}\|\boldsymbol{s}-\boldsymbol{c}\|_{2}^{2} \\
& =\underset{\boldsymbol{s} \in \mathcal{A}}{\arg \min } \frac{1}{2}\left\|\boldsymbol{s}-\left(\boldsymbol{c}-\dot{\mathbf{M}} \boldsymbol{q}_{1}-\ddot{\mathbf{M}}^{*} \boldsymbol{q}_{2}\right)\right\|_{2}^{2} .
\end{aligned}
$$

Therefore, $\boldsymbol{s}^{*}\left(\boldsymbol{q}_{1}, \boldsymbol{q}_{2}\right)$ is the orthogonal projection of $\boldsymbol{z}=\boldsymbol{c}-\dot{\mathbf{M}} \boldsymbol{q}_{1}-\ddot{\mathbf{M}}^{*} \boldsymbol{q}_{2}$ onto $\mathcal{A}$. Since $\mathcal{A}$ is not empty, $\boldsymbol{A} \boldsymbol{A}^{+} \boldsymbol{v}=\boldsymbol{v}$, and the set $\mathcal{A}=\left\{\boldsymbol{s} \in \mathbb{R}^{n \cdot d}, \boldsymbol{A s}=\boldsymbol{v}\right\}$ can be decomposed as

$$
\mathcal{A}=\boldsymbol{A}^{+} \boldsymbol{v}+\operatorname{ker}(\boldsymbol{A}) .
$$

The vector $\boldsymbol{z}-\boldsymbol{s}^{*}\left(\boldsymbol{q}_{1}, \boldsymbol{q}_{2}\right)$ is orthogonal to $\mathcal{A}$, it therefore belongs to $\operatorname{ker}(\boldsymbol{A})^{\perp}=\operatorname{im}\left(\boldsymbol{A}^{*}\right)$. Hence $\boldsymbol{s}^{*}\left(\boldsymbol{q}_{1}, \boldsymbol{q}_{2}\right)=\boldsymbol{z}+\boldsymbol{A}^{*} \boldsymbol{\lambda}$ for some $\lambda$ such that:

$$
\boldsymbol{A}\left(\boldsymbol{z}+\boldsymbol{A}^{*} \boldsymbol{\lambda}\right)=\boldsymbol{v}
$$

This leads to $\boldsymbol{\lambda}=\left(\boldsymbol{A} \boldsymbol{A}^{*}\right)^{-1}(\boldsymbol{v}-\boldsymbol{A} \boldsymbol{z})$. We finally get

$$
\boldsymbol{s}^{*}\left(\boldsymbol{q}_{1}, \boldsymbol{q}_{2}\right)=\boldsymbol{z}+\boldsymbol{A}^{*}\left(\boldsymbol{A} \boldsymbol{A}^{*}\right)^{-1}(\boldsymbol{v}-\boldsymbol{A} \boldsymbol{z}),
$$

ending the proof. 


\section{APPENDIX D \\ PROOF OF THEOREM 1.}

Let us first recall that the relative interior of a convex set $C \operatorname{ri}(C)$ is the interior of $C$ relative to the affine hull of $C$ [29]. The analysis proposed to prove Theorem 1 closely follows ideas proposed in [31,54-56]. We will need two results. The first one is a duality result from [55].

Proposition 8. Let $f: \mathbb{R}^{m} \rightarrow \mathbb{R} \cup\{\infty\}$ and $g: \mathbb{R}^{n} \rightarrow \mathbb{R} \cup\{\infty\}$ denote two closed convex functions, and $\boldsymbol{A} \in \mathbb{R}^{m \times n}$ denote a matrix. Assume that $g$ is $\sigma$-strongly convex [29] and that $\operatorname{Ari}(\operatorname{dom}(f)) \cap \operatorname{ri}(\operatorname{dom}(g)) \neq \emptyset$.

Let $p(\boldsymbol{x})=f(\boldsymbol{A} \boldsymbol{x})+g(\boldsymbol{x})$ and $d(\boldsymbol{y})=-g^{*}\left(\boldsymbol{A}^{*} \boldsymbol{y}\right)-f^{*}(\boldsymbol{y})$. Let $\boldsymbol{x}^{*}$ be the unique minimizer of $p$ and $\boldsymbol{y}^{*}$ be any minimizer of $d$.

Then $g^{*}$ is differentiable with $\frac{1}{\sigma}$ Lipschitz-continuous gradient. Moreover, by letting $\boldsymbol{x}(\boldsymbol{y})=\nabla g^{*}\left(-\boldsymbol{A}^{*} \boldsymbol{y}\right)$ :

$$
\left\|\boldsymbol{x}(\boldsymbol{y})-\boldsymbol{x}^{*}\right\|_{2}^{2} \leq \frac{2}{\sigma}\left(d(\boldsymbol{y})-d\left(\boldsymbol{y}^{*}\right)\right)
$$

The second ingredient is the standard convergence rate for accelerated proximal gradient descents given in [31, Theorem. 4.4].

Proposition 9. Under the same assumptions as Proposition 8, consider Algorithm 9.

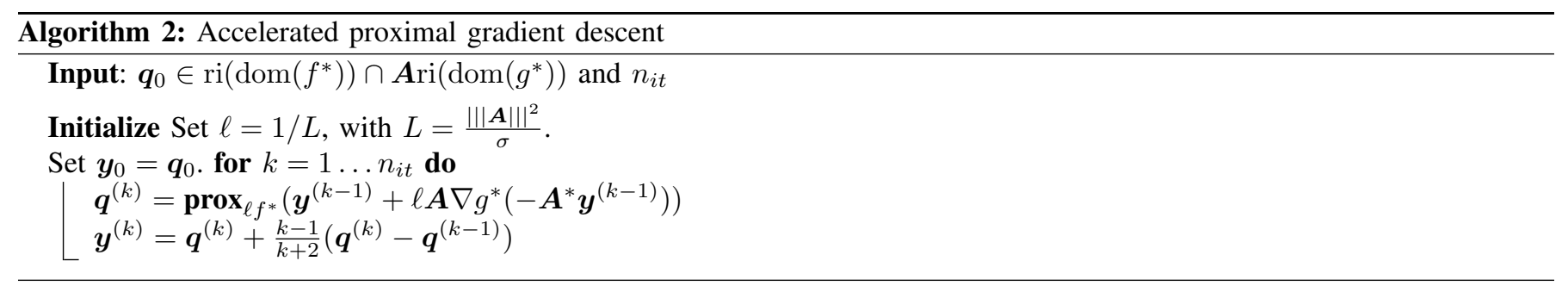

Then $\left\|\boldsymbol{y}^{\left(n_{i t}\right)}-\boldsymbol{y}^{*}\right\|_{2}^{2}=\mathcal{O}\left(\frac{\|\boldsymbol{A}\| \|^{2}}{\sigma \cdot n_{i t}^{2}}\right)$.

To conclude, it suffices to set $g(\boldsymbol{s})=\frac{1}{2}\|\boldsymbol{s}-\boldsymbol{c}\|_{2}^{2}, f\left(\boldsymbol{q}_{1}, \boldsymbol{q}_{2}\right)=\imath_{\boldsymbol{B}_{\alpha}}\left(\boldsymbol{q}_{1}\right)+\imath_{\boldsymbol{B}_{\beta}}\left(\boldsymbol{q}_{2}\right)$ and $\boldsymbol{A}=\left(\begin{array}{c}\dot{\mathbf{M}} \\ \ddot{\mathbf{M}}\end{array}\right)$. By doing so, the projection problem rewrites $\min _{\boldsymbol{s} \in \mathbb{R}^{n \dot{d}}} p(\boldsymbol{s})=f(A \boldsymbol{s})+g(\boldsymbol{s})$. Its dual problem (8) can be rewritten more compactly as $\min _{\boldsymbol{q}=\left(\boldsymbol{q}_{1}, \boldsymbol{q}_{2}\right) \in \mathbb{R}^{n \dot{d}} \times \mathbb{R}^{n \dot{d}}} d(\boldsymbol{q})=$ $g^{*}\left(-\boldsymbol{A}^{*} \boldsymbol{q}\right)+f^{*}(\boldsymbol{q})$. Note that function $g$ is 1 -strongly convex. Therefore, Algorithm 9 can be used to minimize $d$, ensuring a convergence rate in $\mathcal{O}\left(\frac{L}{k^{2}}\right)$ on the function values $d\left(\boldsymbol{y}^{(k)}\right)$, where $L=\|\boldsymbol{A}\| \|^{2}$. It then suffices to use Proposition 8 to obtain a convergence rate on the distance to the solution $\left\|s^{(k)}-s^{*}\right\|_{2}^{2}$. This ends the proof of Theorem 1.

\section{REFERENCES}

[1] J. Bigot, C. Boyer, and P. Weiss, “An analysis of block sampling strategies in compressed sensing," IEEE Trans. Inf. Theory, p. To appear, 2016.

[2] N. Chauffert, P. Ciuciu, J. Kahn, and P. Weiss, "Variable density sampling with continuous trajectories. Application to MRI," SIAM Journal on Imaging Science, vol. 7, no. 4, pp. 1962-1992, 2014.

[3] C. Boyer, J. Bigot, and P. Weiss, "Compressed sensing with structured sparsity and structured acquisition," arXiv preprint arXiv:1505.01619, 2015.

[4] J. Unnikrishnan and M. Vetterli, "Sampling high-dimensional bandlimited fields on low-dimensional manifolds," Information Theory, IEEE Transactions on, vol. 59, no. 4, pp. 2103-2127, 2013.

[5] K. Gröchenig, J. L. Romero, J. Unnikrishnan, and M. Vetterli, "On minimal trajectories for mobile sampling of bandlimited fields," Applied and Computational Harmonic Analysis, 2014.

[6] G. Puy, P. Vandergheynst, and Y. Wiaux, "On variable density compressive sampling," IEEE Signal Processing Letters, vol. 18, no. 10, pp. 595-598, 2011.

[7] F. Krahmer and R. Ward, "Beyond incoherence: stable and robust sampling strategies for compressive imaging." preprint, 2012.

[8] P. T. Gurney, B. A. Hargreaves, and D. G. Nishimura, "Design and analysis of a practical 3D cones trajectory," Magn. Reson. Med., vol. 55, no. 3, pp. 575-582, 2006.

[9] J. G. Pipe and N. R. Zwart, "Spiral trajectory design: A flexible numerical algorithm and base analytical equations," Magn. Reson. Med., vol. 71, no. 1, pp. 278-285, 2014.

[10] M. Lustig, D. L. Donoho, and J. M. Pauly, "Sparse MRI: The application of compressed sensing for rapid MR imaging," Magn. Reson. Med., vol. 58, pp. 1182-1195, Dec. 2007.

[11] L. Feng, R. Grimm, K. T. Block, H. Chandarana, S. Kim, J. Xu, L. Axel, D. K. Sodickson, and R. Otazo, "Golden-angle radial sparse parallel mri: Combination of compressed sensing, parallel imaging, and golden-angle radial sampling for fast and flexible dynamic volumetric mri," Magn. Reson. Med., vol. 72, no. 3, pp. 707-717, 2014.

[12] M. Lustig, J. H. Lee, D. L. Donoho, and J. M. Pauly, "Faster imaging with randomly perturbed, under-sampled spirals and 11 reconstruction," in Proceedings of the 13th annual meeting of ISMRM, Miami Beach, p. 685, 2005.

[13] D. C. Noll, "Multishot rosette trajectories for spectrally selective MR imaging," IEEE Trans. Med. Imag., vol. 16, no. 4, pp. 372-377, 1997.

[14] Y. Shu, S. J. Riederer, and M. A. Bernstein, "Three-dimensional mri with an undersampled spherical shells trajectory," Magn. Reson. Med., vol. 56, no. 3, pp. 553-562, 2006.

[15] M. Lustig, S. J. Kim, and J. M. Pauly, "A fast method for designing time-optimal gradient waveforms for arbitrary k-space trajectories," IEEE Trans. Med. Imag., vol. 27, no. 6, pp. 866-873, 2008.

[16] O. P. Simonetti, J. L. Duerk, and V. Chankong, "An optimal design method for magnetic resonance imaging gradient waveforms," IEEE Trans. Med. Imag., vol. 12, no. 2, pp. 350-360, 1993. 
[17] B. A. Hargreaves, D. G. Nishimura, and S. M. Conolly, "Time-optimal multidimensional gradient waveform design for rapid imaging," Magn. Reson. Med., vol. 51, no. 1, pp. 81-92, 2004

[18] M. Davids, M. Ruttorf, F. Zoellner, and L. Schad, "Fast and robust design of time-optimal $k$-space trajectories in MRI," IEEE Trans. Med. Imag., vol. 34, no. 2, pp. 564-577, 2015.

[19] B. Adcock, A. Hansen, C. Poon, and B. Roman, "Breaking the coherence barrier: asymptotic incoherence and asymptotic sparsity in compressed sensing," tech. rep., University of Cambridge, Cambridge, UK, 2013.

[20] N. Chauffert, P. Ciuciu, J. Kahn, and P. Weiss, "Travelling salesman-based compressive sampling," in Proc. of 10th SampTA conference, (Bremen, Germany), pp. 509-511, July 2013.

[21] N. Chauffert, P. Ciuciu, P. Weiss, and F. Gamboa, "From variable density sampling to continuous sampling using Markov chains," in Proc. of 10th SampTA conference, (Bremen, Germany), pp. 200-203, July 2013.

[22] H. Wang, X. Wang, Y. Zhou, Y. Chang, and Y. Wang, "Smoothed random-like trajectory for compressed sensing MRI," in Proc. of the 34th annual IEEE EMBC, pp. 404-407, 2012.

[23] R. M. Willett., "Errata: Sampling trajectories for sparse image recovery," note, Duke University, 2011.

[24] N. Chauffert, Compressed sensing along physically plausible sampling trajectories. PhD thesis, Université Paris-Sud, Orsay, France, Sep. 2015.

[25] K. Majewski, O. Heid, and T. Kluge, "MRI pulse sequence design with first-order gradient moment nulling in arbitrary directions by solving a polynomial program," IEEE Trans. Med. Imag., vol. 29, no. 6, pp. 1252-1259, 2010.

[26] N. Chauffert, P. Ciuciu, and P. Weiss, "Variable density compressed sensing in MRI. Theoretical vs. heuristic sampling strategies," in Proc. of 10th IEEE ISBI conference, (San Francisco, USA), pp. 298-301, Apr. 2013.

[27] C. Villani, Optimal transport: old and new, vol. 338. Springer, 2008.

[28] Y. Nesterov, "Smooth minimization of non-smooth functions," Math. Program., vol. 103, pp. 127-152, May 2005.

[29] J.-B. Hiriart-Urruty and C. Lemaréchal, Convex Analysis and Minimization Algorithms I: Part 1: Fundamentals, vol. 305. Springer, 1996.

[30] Y. Nesterov, "A method of solving a convex programming problem with convergence rate $\mathcal{O}\left(1 / k^{2}\right)$," in Soviet Mathematics Doklady, vol. 27, pp. 372-376, 1983.

[31] A. Beck and M. Teboulle, "Gradient-based algorithms with applications to signal recovery," Convex Optimization in Signal Processing and Communications, 2009.

[32] M. P. Sampat, Z. Wang, S. Gupta, A. C. Bovik, and M. K. Markey, "Complex wavelet structural similarity: A new image similarity index," Image Processing, IEEE Transactions on, vol. 18, no. 11, pp. 2385-2401, 2009.

[33] E. Candès, J. Romberg, and T. Tao, "Robust uncertainty principles: exact signal reconstruction from highly incomplete frequency information," IEEE Trans. Inf. Theory, vol. 52, no. 2, pp. 489-509, 2006.

[34] J. Keiner, S. Kunis, and D. Potts, "Using nfft 3-a software library for various nonequispaced fast fourier transforms," ACM Transactions on Mathematical Software (TOMS), vol. 36, no. 4, p. 19, 2009.

[35] M. Guerquin-Kern, L. Lejeune, K. P. Pruessmann, and M. Unser, "Realistic analytical phantoms for parallel magnetic resonance imaging," Medical Imaging, IEEE Transactions on, vol. 31, no. 3, pp. 626-636, 2012.

[36] A. Beck and M. Teboulle, "A fast iterative shrinkage-thresholding algorithm for linear inverse problems," SIAM Journal on Imaging Sciences, vol. 2, no. 1, pp. 183-202, 2009.

[37] S. Chen and D. Donoho, "Basis pursuit," in Signals, Systems and Computers, 1994. 1994 Conference Record of the Twenty-Eighth Asilomar Conference on, vol. 1, pp. 41-44, IEEE, 1994.

[38] E. Van Den Berg and M. P. Friedlander, "Probing the pareto frontier for basis pursuit solutions," SIAM Journal on Scientific Computing, vol. 31, no. 2, pp. 890-912, 2008.

[39] E. Van den Berg and M. P. Friedlander, "Sparse optimization with least-squares constraints," SIAM Journal on Optimization, vol. 21, no. 4, pp. 1201-1229, 2011.

[40] A. Florescu, E. Chouzenoux, J.-C. Pesquet, P. Ciuciu, and S. Ciochina, "A majorize-minimize memory gradient method for complex-valued inverse problems," Signal Processing, vol. 103, pp. 285-295, 2014.

[41] Y. Huang, J. Paisley, Q. Lin, X. Ding, X. Fu, and X.-P. Zhang, "Bayesian nonparametric dictionary learning for compressed sensing mri," Image Processing, IEEE Transactions on, vol. 23, no. 12, pp. 5007-5019, 2014.

[42] C. Boyer, N. Chauffert, P. Ciuciu, J. Kahn, and P. Weiss, "On the generation of sampling schemes for magnetic resonance imaging," submitted to SIAM Imaging Science, ITAV/CNRS Toulouse and CEA/NeuroSpin, Saclay, Jan. 2016.

[43] K. P. Pruessmann, M. Weiger, M. B. Scheidegger, and P. Boesiger, "SENSE: sensitivity encoding for fast MRI," Magn. Reson. Med., vol. 42, pp. 952-962, Jul. 1999.

[44] M. A. Griswold, P. M. Jakob, R. M. Heidemann, M. Nittka, V. Jellus, J. Wang, B. Kiefer, and A. Haase, "Generalized autocalibrating partially parallel acquisitions GRAPPA," Magn. Reson. Med., vol. 47, pp. 1202-1210, Jun. 2002.

[45] D. A. Feinberg, S. Moeller, S. M. Smith, E. Auerbach, S. Ramanna, M. Gunther, M. F. Glasser, K. L. Miller, K. Ugurbil, and E. Yacoub, "Multiplexed echo planar imaging for sub-second whole brain FMRI and fast diffusion imaging," PloS one, vol. 5, no. 12, p. e15710, 2010.

[46] N. Chauffert, P. Ciuciu, J. Kahn, and P. Weiss, "A projection algorithm on measures sets," to appear in Constructive Approximation, Mar. 2016.

[47] G. Varoquaux and R. C. Craddock, "Learning and comparing functional connectomes across subjects," NeuroImage, vol. 80, pp. 405-415, 2013.

[48] P. Ciuciu, P. Abry, and B. J. He, "Interplay between functional connectivity and scale-free dynamics in intrinsic fMRI networks," NeuroImage, vol. 95, pp. 248-263, 2014

[49] T. Vincent, L. Risser, and P. Ciuciu, "Spatially adaptive mixture modeling for analysis of fMRI time series," Medical Imaging, IEEE Transactions on, vol. 29, no. 4, pp. 1059-1074, 2010.

[50] L. Chaari, T. Vincent, F. Forbes, M. Dojat, and P. Ciuciu, "Fast joint detection-estimation of evoked brain activity in event-related fMRI using a variational approach," IEEE Trans. Med. Imag., vol. 32, pp. 821-837, May 2013.

[51] F. Pedregosa, M. Eickenberg, P. Ciuciu, B. Thirion, and A. Gramfort, "Data-driven HRF estimation for encoding and decoding models," NeuroImage, vol. 104, pp. 209-220, 2015.

[52] N. Chauffert, P. Weiss, M. Boucher, S. Mériaux, and P. Ciuciu, "Variable density sampling based on physically plausible gradient waveform. Application to 3D MRI angiography," in 12th Proc. IEEE ISBI, (New-York, NY), pp. 298-301, Apr. 2015.

[53] R. T. Rockafellar, Convex analysis. No. 28, Princeton university press, 1997.

[54] P. Weiss, L. Blanc-Féraud, and G. Aubert, "Efficient schemes for total variation minimization under constraints in image processing," SIAM journal on Scientific Computing, vol. 31, no. 3, pp. 2047-2080, 2009.

[55] C. Boyer, P. Weiss, and J. Bigot, "An algorithm for variable density sampling with block-constrained acquisition," SIAM Journal on Imaging Sciences, vol. 7, no. 2, pp. 1080-1107, 2014.

[56] A. Beck and M. Teboulle, "A fast dual proximal gradient algorithm for convex minimization and applications," Operations Research Letters, vol. 42, no. 1, pp. 1-6, 2014. 